\title{
Approximate Best-Response Dynamics in Random
}

\section{Interference Games}

\author{
Ilai Bistritz, Student Member, IEEE, and Amir Leshem, Senior Member, IEEE
}

\begin{abstract}
In this paper, we develop a novel approach to the convergence of Best-Response Dynamics for the family of interference games. Interference games represent the fundamental resource allocation conflict between users of the radio spectrum. In contrast to congestion games, interference games are generally not potential games. Therefore, proving the convergence of the best-response dynamics to a Nash equilibrium in these games requires new techniques. We suggest a model for random interference games, based on the long term fading governed by the players' geometry. Our goal is to prove convergence of the approximate best-response dynamics with high probability with respect to the randomized game. We embrace the asynchronous model in which the acting player is chosen at each stage at random. In our approximate best-response dynamics, the action of a deviating player is chosen at random among all the approximately best ones. We show that with high probability, with respect to the players' geometry and asymptotically with the number of players, each action increases the expected social-welfare (sum of achievable rates). Hence, the induced sum-rate process is a submartingale. Based on the Martingale Convergence Theorem, we prove convergence of the strategy profile to an approximate Nash equilibrium with good performance for asymptotically almost all interference games. We use the Markovity of the induced sum-rate process to provide probabilistic bounds on the convergence time. Finally, we demonstrate our results in simulated examples.
\end{abstract}

\section{Index Terms}

Ad-Hoc Networks, Best-Response (BR) Dynamics, Interference Channels, Martingales, Random Games

\section{INTRODUCTION}

A PPLICATIONS of game theory to networks in general and wireless networks in particular is an active field of research (see [2]-[6]). By modeling each user or node on a wireless network (e.g. mobile device, access point, etc.) as a player, many conflicts and dynamics that occur in wireless networks can be modeled as a game and analyzed using game theoretic tools. Beyond pure analytic benefits, the game theoretic tools can contribute to the development of distributed algorithms, since each player acts independently and responds to his environment. The outcome of the interaction between these independent nodes is predicted by game-theoretic solution concepts. The best known solution concept is the Nash equilibrium (NE, see [7]).

Ilai Bistritz is with the department of Electrical Engineering-Systems, Tel-Aviv University, Israel, e-mail: ilaibist@gmail.com.

Amir Leshem is with the Faculty of Engineering, Bar-Ilan University, Ramat-Gan, Israel, e-mail: leshema@eng.biu.ac.il.

This research was supported by the Israel Science Foundation, under grant 903/2013, and partially supported by the Israeli ministry of science grant no. 204354.

Parts of this paper were presented at the 55th IEEE Conference on Decision and Control [1]. 
An analysis of the NE points of a game can indicate how good the outcome of the interaction is, in terms of designer-defined performance.

When attempting to apply game theoretic tools for engineering and algorithmic purposes, the question of convergence to an equilibrium is crucial, and goes beyond simply justifying that some equilibrium notion can predict the outcome of a game well. No equilibrium can have a practical meaning without being a natural stable point for some dynamics that are sufficiently easy to implement.

Best-Response Dynamics (BR Dynamics) are well known in game theory. In BR dynamics each player plays his best response to the current actions of the other players. This means that the NE is a natural stable point for BR dynamics. Due to their distributed nature and simple implementation, they were widely applied for various tasks over networks [8]-[10]. Simple as they are, they are not guaranteed to converge.

In [11], the authors presented the concept of sink equilibria, which is a set of strategy profiles that pure BR dynamics cannot escape from, once they attain it. While convergence of randomized pure BR dynamics to a sink equilibrium is guaranteed, the result in general is an oscillating dynamics which is impractical and undesirable in many applications. Furthermore, without any additional information it is impossible to analyze the performance of the resulting equilibria or the convergence rate.

A necessary condition for BR dynamics to converge to a pure NE (PNE) from any initial strategy profile is that there exists a path induced by BR moves that connects the initial strategy to a PNE. A game with such a property is called a weakly acyclic game [12]. In some scenarios, this condition can be considered sufficient, since it has been shown that randomized BR dynamics converge almost surely in weakly acyclic games [13] despite the fact that a weakly acyclic game may contain cycles of BR moves.

While the convergence of BR dynamics in weakly acyclic games is indeed encouraging, the main problem is that reliably checking weak acyclicity is computationally intractable in the worst case [14]. Other than that, it still does not provide indications as to the rate of convergence to an equilibrium and cannot guarantee, in general, the quality of the resulting NE.

A special case of weak acyclic games where more can be said about convergence is potential games [15]. Potential games are games in which there exists a function, known as the potential function, such that for each player, any change in the utility causes the same change (numerically or with the same sign) in the potential function. Potential games exhibit at least one pure NE, and this NE maximizes the potential function. Furthermore, a sequential BR dynamics can easily be shown to converge to an NE. These are very desirable properties, because if the potential function has something to do with the performance required, they indicate that the convergence is to a good NE [16]. Motivated by these appealing properties, many game formulations distributed algorithms in networks have used potential games (see [?], [17]-[21]).

Congestion games are a general model for resource allocation games, and are a special class of potential games. In a congestion game, there are some available routes from a source to a destination, and each player's strategy is to choose his own routes from the set of all routes. The utility function for the player is the sum of the delays in his chosen routes, where the delay in a route is a function that decreases with the number of players who chose this route. It has been shown that convergence to a pure $\mathrm{NE}$ in congestion games can be very slow [22]. In fact, there are examples of games and initial strategies where the shortest path to an equilibrium in the BR dynamics is exponentially long in terms of the number of players. Fortunately, if 
one is willing to settle for an approximate NE, convergence can be a lot faster [23].

While congestion games have successfully been used to model resource allocation in networks [24]-[26], they represent a high-layered approach and are not suited to modeling similar scenarios in the physical layer. To the best of our knowledge, the only effort that has been made to generalize congestion games to wireless networks scenarios is [27]. The authors added an interaction graph that defines which player is interacting with whom, and so captures some notion of spatial geometry. Although constituting a step forward, convergence to an NE was based again on a potential function, which was shown only for special cases of interaction graphs. Moreover, the interaction graph does not really capture the continuous nature of the interference in a wireless network. From a more general perspective, the authors in [28], [29] studied the properties of games that are near-potential in terms of their utility functions, and suggested dynamics that converge to an approximate NE for such games. The accuracy of the approximate NE is dependent on the distance of the game from a potential game.

The family of interference games appears naturally when modeling the capacity of wireless networks and frequency allocation problems. Here we aim to generalize the desired convergence properties of congestion games to these games. This is not an easy task. First, interference games tend not to be potential games, and so BR dynamics convergence, if occurs, should be established using other techniques. Even worse, there are examples of non-weakly acyclic interference games in which the BR dynamics fail to converge [5]. Our goal is to overcome these obstacles by analyzing an approximate BR convergence for a random interference game, where the channel gains represent the long term fading determined by the players' locations. We show that, asymptotically in the number of players, almost all interference games do have converging approximate BR dynamics.

We analyze the probability for convergence asymptotically in the number of players. The area of the region where players are located is kept constant. The large number of players limit is toward denser networks with a large number of devices in a given area, and has special significance due the recent growing interest in large-scale networks. Nevertheless, this limit has analytical advantages (for example, see [30]). Two closely related branches of game theory that exploit this limit regularly are mean-field games and evolutionary games. In mean-field games, each player is acting in response to an aggregated state created by the choices of the mass [31], [32]. Our approach is essentially different from the above approach since in our game the influence of each player on specific other players can be fatal, and not negligible. In evolutionary games the analyzed dynamics are of the relative parts of the population that chose each strategy [33]-[35]. This requires that players will be anonymous only the number of players choosing a specific strategy matters. In contrast, in interference games each player has a unique characteristic that stems from his position in space. In this sense, our results can be thought of as an alternative approach to large-scale games that maintains the individuality and uniqueness of each player. Another essential issue is that the above approaches require a large number of players to be considered valid. In our approach, the percentage of games for which the results are valid grows as the number of players grows, and can be bounded from below for each finite number of players.

Together with the assumption of fixed players' locations during the dynamics, our approach is best suited for large scale communication scenarios such as the internet of things (IoT), wireless sensor and actuator networks (WSAN), cellular backbone and infrastructure with picocells and femtocells, smart homes and cities, hotspots and networks with pedestrian mobility.

The rest of this paper is organized as follows. In Section II we define interference games and formulate the random 
interference game and our system model. In section III we define our Approximate BR Dynamics, and in Section IV we prove that these dynamics induce a sum-rate process that is a submartingale. This establishes the convergence of the dynamics to an approximate NE based on the Martingale Convergence Theorem. Section V provides an analysis of the convergence time of the dynamics, and Section VI presents simulations that confirm our results. Finally, we draw conclusions in section VII.

This paper provides full proofs for the results presented in [1], along with a much more detailed discussion. Additionally, this paper generalizes several assumptions made in [1] (like constant load, omnidirectional transmission and the scheduling) and adds a convergence time analysis. The numerical results have also been extended.

\section{INTERFERENCE GAMES}

In this section we introduce interference games. We define a random model for interference games and specify our system model in the limit of a large number of players.

The fundamental conflict that arises from interference in the physical layer has become a source for many game-theoretic formulations (see [3], [5], [6], [36]-[39] and the references therein), many of them use the transmission power as the strategy of each player. Here we aim to define a general framework for channel selection games in the interference channel, and argue that in this form they are a natural generalization to the well-known congestion games. All the games we deal with have a finite number of players and finite strategy spaces.

We start by introducing congestion games, which are a special case of potential games which is useful for modeling resource allocation scenarios.

Throughout this paper, we use the standard game-theoretic notation where $\boldsymbol{a}_{-n}=\left(a_{1}, \ldots, a_{n-1}, a_{n+1}, \ldots, a_{N}\right)$ denotes the strategy profile of all player $n$ 's opponents, and $A_{-n}=A_{1} \times \ldots \times A_{n-1} \times A_{n+1} \times \ldots \times A_{N}$ is the space of all those strategy profiles.

Definition 1 (Ordinal Potential Game). A normal-form game $G=<\mathcal{N},\left\{A_{n}\right\}_{n \in \mathcal{N}},\left\{u_{n}\right\}_{n \in \mathcal{N}}>$ is an ordinal potential game if there exists a function $\Phi: A_{1} \times \ldots \times A_{N} \rightarrow \mathbb{R}$ such that for each $n \in \mathcal{N}$ and for all $a_{n}, a_{n}^{\prime} \in A_{n}$ and $\mathbf{a}_{-n} \in A_{-n}$, $\Phi\left(a_{n}, \mathbf{a}_{-n}\right)-\Phi\left(a_{n}^{\prime}, \mathbf{a}_{-n}\right)>0$ if and only if $u_{n}\left(a_{n}, \mathbf{a}_{-n}\right)-u_{n}\left(a_{n}^{\prime}, \mathbf{a}_{-n}\right)>0$.

A potential game has at least one pure Nash equilibrium [40]. If the game has not yet reached an equilibrium, then the turn of a deviating player will arrive eventually and his action will increase the potential function. After enough time, the potential function will reach a maximum and no player will benefit from deviating, so convergence to an NE occurs.

Definition 2 (Congestion Game). A game with $N$ players and a set of congestible elements $E$ is called a congestion game if for each $n, a_{n} \subseteq E$ for each $a_{n} \in A_{n}$ and the utility of player $n$ is in the form $u_{n}(\mathbf{a})=\sum_{e \in a_{n}} c_{e}\left(x_{e}\right)$, where each $c_{e}$ is a positive monotonically decreasing function and $x_{e}=\left|\left\{n \mid e \in a_{n}\right\}\right|$.

Next, we define interference games.

Definition 3 (Interference Game). An interference game is a game with a set $\mathcal{N}$ of $N$ players (each is a transceiver), where each player $n$ has another player $d(n)$ as his destination, and a set $\mathcal{K}$ of $K$ channels. Each player's action is a subset of channels, so for each $n, a_{n} \subseteq \mathcal{K}$ for each $a_{n} \in A_{n}$. The utility of each player is in the form $u_{n}(\mathbf{a})=\sum_{k \in a_{n}} R_{k}\left(I_{k}(\mathbf{a})\right)$, 
where each $R_{k}$ is a positive monotonically decreasing function and $I_{k}(\mathbf{a})=\sum_{\left\{m \mid k \in a_{m}\right\}} g_{m, d(n)} P_{m}$. The coefficient $g_{m, d(n)}$ is the channel gain between player $m$ to the destination of player $n$, and $P_{m}$ is the transmission power of player $m$.

We emphasize that the definition above is general and allows each player to choose multiple channels. This shows the analogy between interference games and congestion games. However, for the sake of simplicity, we focus in this paper on the case where each player chooses a single channel among the set $\mathcal{K}$.

Interference games are essentially different from congestion games. They generalize them in the sense that in an interference game, the effect of some player $m$ on player $n$ is different for each different choice of $n, m$. Although higher numbers of players produce higher interference on average, the exact interference is a weighted sum of the channel gains between the involved players. This fact, which stems directly from the geometry of the players in space, has major implications for the convergence of BR dynamics to a NE.

Interference games tend not to be potential games (see the example in [1]), and in fact most of them are not 1 . Consequently, BR convergence is not guaranteed. Furthermore, one can construct an interference game that is not weakly acyclic, so there is no hope for BR dynamics to converge in all interference games [5]. The same is true for an approximate NE with a small enough epsilon. Alternatively, for any epsilon there is an interference game with no such approximate NE. Nevertheless, we are interested in typical scenarios and the behavior of the majority of interference scenarios. Theoretically, each interference game realization, i.e. a set of channel gains, may have different equilibria and convergence properties, and there is an infinite number of such realizations. We use the following random model to show that, asymptotically in the number of players, almost all of them do have converging (approximate) BR dynamics.

\section{A. Random Interference Game}

It is a common practice in wireless networks to model the channel gains as random variables with some appropriate distribution. Our goal is to analyze the probability that a certain interference game, drawn at random according to this distribution, will have converging approximate BR dynamics. In this subsection, we propose a random model for interference games. It is by no means the only reasonable random model, and other scenarios may require different models.

Our channel gains distribution is dictated by the random player locations. We assume that the players' locations are generated uniformly and independently at random in some subregion of the real plane, $\mathcal{D} \subseteq \mathbb{R}^{2}$, with area $|\mathcal{D}|$. Each player as a transmitter has some other player's receiver as his destination. We also explore the effect of beamforming on the convergence.

Definition 4 (Channel Gains). Assume that each transceiver is capable of transmitting a beam with angle $\theta_{T}$ and receiving a beam with angle $\theta_{R}$, with some arbitrary orientation. The channel gains are determined directly from the players' locations and the beamforming angles, so the channel gain between player $n$ to the destination of player $m$ is defined by

$$
g_{n, d(m)}=\frac{G}{r_{n, d(m)}^{\alpha}} I_{d}\left\{\left|\theta_{n, d(m)}\right| \leq \frac{\theta_{T}}{2}\right\} I_{d}\left\{\left|\theta_{d(m), n}\right| \leq \frac{\theta_{R}}{2}\right\}
$$

${ }^{1}$ This can be verified by detecting BR cycles. 
where $I_{d}$ is the indicator function, $r_{n, d(m)}$ is the Euclidean distance between player $n$ and player $m$ 's destination, $\alpha$ is the path-loss exponent and $G=\left(\frac{\nu}{4 \pi}\right)^{\alpha}$, where $\nu$ is the wavelength. The angles $\theta_{n, d(m)}$ and $\theta_{d(m), n}$ are defined with respect to the beamforming angle bisectors of players $n$ and $d(m)$, respectively.

The beamforming angels are chosen such that the interference (transmitted or received) outside the beam can be neglected (e.g., $10 \mathrm{~dB}$ beamwidth). This type of beamforming is natural for large scale networks, since the number of interferers tend to be larger than the degrees of freedom of the multiple-input and multiple-output (MIMO) transceiver, so zero forcing is impractical. Acquiring the required channel state information in a distributed network is also a major issue. Substituting $\theta_{T}=\theta_{R}=2 \pi$ in (1) results in the special case of omnidirectional transmission and reception.

Our model does not include instantaneous small scale-fading. This may be considered a result of averaging the channel gains over multiple coherence times, which occurs if our dynamics have a duration that is significantly larger than the coherence-time of the wireless channel. From a system perspective this is very natural, since each player needs to sense the interference in each frequency band in order to choose his new channel; hence channel switching typically requires a longer time scale than the coherence time. Additionally, the assumption of constant locations is better suited to a scenario with mobility rates that are much slower than the convergence time of the dynamics.

We use the achievable rate when interference is treated as noise as our utility function

$$
u_{n}(\mathbf{a})=R_{n}(\mathbf{a})=R_{n}\left(I_{n}(\mathbf{a})\right)=\log _{2}\left(1+\frac{g_{n, d(n)} P_{n}}{N_{0}+I_{n}(\mathbf{a})}\right)
$$

where $P_{n}$ is player $n$ 's transmission power, which is limited by $P_{n} \leq P_{\max }$ for all $n$, and $N_{0}$ is the Gaussian noise variance. We emphasize that this utility is of player $n$ and does not reflect on the utility of player $d(n)$. The utility of player $m=d(n)$ is measured by his achievable rate in the transmission to $d(m)$. Observe that the social welfare of an interference game is the sum of the achievable rates, which is a desirable performance measure for a channel allocation algorithm. We assume that each player can sense the exact interference in each of the $K$ channels and adapts his transmission scheme accordingly. This sensing capability allows each player to compute his utility function in each channel, provided that other players stay in their current channel.

\section{B. System Model}

As in ad-hoc networks, in our network each player has another player as his destination. This need not be his final destination, but just the next hop. We assume that these destinations are constant over the duration of our dynamics, and that each formed link is constantly active (although that not necessarily with a continuous transmission).

Our analysis is asymptotic in $N$, the number of players. A similar approach was taken in [37], but for analyzing the structure of Nash equilibria in frequency-selective interference games. The goal of this subsection is to define how the system parameters behave in the limit where $N$ approaches infinity. These parameters are the number of channels, the transmission powers and the area of the players' region. Note that since each player demands some specific target rate, the transmission power of each player is dictated by his distance from his destination and vice versa. A parameter that is fixed with respect to $N$ will be termed "constant". 
We assume that the region $\mathcal{D} \subseteq \mathbb{R}^{2}$, where the players are located, has area $|\mathcal{D}|=\frac{1}{\lambda}$ for some $\lambda>0$, so it is fixed with respect to $N$. This yields an increasing players' density as $N \rightarrow \infty$. This is in the spirit of large scale networks such as the IoT, sensor and actuator networks, cellular networks with femtocells, smart homes and cities and others.

Now we have to decide about $K$, the number of channels, and $P_{\max }$, the maximal transmission power. We argue that the only reasonable choice is having both $K \rightarrow \infty$ and $P_{\max } \rightarrow 0$. If only $K \rightarrow \infty$ but $P_{\max }$ is fixed with respect to $N$, then there are a constant (or even an increasing) number, $\frac{N}{K}$, of players per channel on average. A constant $P_{\max }$ allows each player to choose a destination from all the other players in the region $\mathcal{D}$. Hence a player with a destination that is within a constant distance from him will suffer from $\frac{N}{K}-1$ strong interferers. Each of these interferers transmits with a constant power and is within a constant distance away. Consequently, this player will be limited by a negative (in decibels) signal to interference ratio. Now assume instead that $K$ is fixed with respect to $N$ but $P_{\max } \rightarrow 0$. In this case the average number of players per channel, $\frac{N}{K}$, grows to infinity. Since $P_{\max } \rightarrow 0$ forces each player to choose only a nearby player as a destination, each pair of source-destination will be geometrically close (with a decreasing distance with $N$ ). Hence, the growing number of players on a specific channel have to be geometrically separated enough from each other. Otherwise their signal to interference ratio will be negative (in decibels) due to the aggregated interference. Unfortunately, this is not possible, since the region $\mathcal{D}$ has a fixed area with respect to $N$.

Following the argument above we assume that the system load per frequency, defined as the ratio $l_{N}=\frac{N}{K}$, satisfies $l_{N}\left(\frac{\log N}{N}\right)^{\frac{\alpha}{\alpha+2}} \rightarrow 0$ as $N \rightarrow \infty$, so $l_{N}=o\left(\left(\frac{N}{\log N}\right)^{\frac{\alpha}{\alpha+2}}\right)$ (where $\alpha$ is the path-loss exponent). This of course holds for the special case of constant load, i.e., $l_{N}=l$ (presented in [1]), but even for $l_{N}=\sqrt{N}$ for any $\alpha>2$. These assumptions yield an increasing (or at least constant) network wide spectral efficiency in bps per $\mathrm{Hz}$ per square meter. Without loss of generality, we assume that $\frac{N}{K}=l_{N} \geq 1$, since the analysis for all the cases where $N<K$ readily follows from that of the $N=K$ case.

Interestingly, the requirement that $P_{\max } \rightarrow 0$ as $N \rightarrow \infty$ can be also deduced from a broader perspective, that takes into account the routing mechanism applied in the network. In the fundamental work [41], it was shown that if the (final) destinations are chosen arbitrarily in $\mathcal{D}$ then, even if the users' locations, transmission ranges for each hop and the transmission schedules are all centrally optimized, each user will have a vanishing throughput when the number of users grows. This result is valid under the assumptions of stationary users and interference that is treated as noise. Dropping the stationarity assumption, it has indeed been shown that mobility can be exploited to avoid the vanishing throughput for the price of significant delays, but this is far from practice for most applications [42]. Instead of treating the interference as noise, advanced decoding techniques such as interference cancellation or interference alignment can theoretically be applied. Unfortunately, these are essentially impractical in large-scale networks due to the immense channel state information required from each user.

This leads us to assume that the transmission range of each player is limited by $r_{\max }=\sqrt{\frac{\log N+a_{N}}{\pi N}}$ for some constant $r_{0}>0$ and $a_{N} \rightarrow \infty$ as $N \rightarrow \infty$. This transmission range is far from arbitrary in networks with random and uniform players' placement. Define two players as connected if they are within a transmission range from each other. The radius $r_{\max }$ is known to asymptotically guarantee the connectivity of the network [43], and specifically allows a player to choose any one of his nearest neighbors as the next hop. In fact, the throughput increases as the transmission range decreases, and since the network should maintain connectivity, $r_{\max }$ is the ideal transmission range. Indeed, for this choice, we obtain that if the number of hops 
until the final destination, for each player, is constant with respect to $N$, then the throughput per player does not vanish as $N \rightarrow \infty$. If transmit or receive beamforming is used, the transmission range that guarantees asymptotic connectivity remains the same up to a constant, although a special care should be given to edge effects, antenna pattern and the beamforming technique [44]. According to this approach, a player that wishes to transmit packets to a far away player should do so by choosing a wired nearby player as his physical destination. This is possible if wired players are deployed densely enough and the routing algorithm is designed accordingly.

To implement the assumption on the transmission range, we set the maximal transmission power to $P_{\max }=P_{0}\left(\frac{\log N}{N}\right){ }^{\frac{\alpha}{2}}$ for some $P_{0}>0$. If $r_{n, d(n)} \leq \sqrt{\frac{\log N}{N}}$ then $g_{n, d(n)} \geq G\left(\frac{N}{\log N}\right)^{\frac{\alpha}{2}}$ and so $P_{\max }=P_{0}\left(\frac{\log N}{N}\right)^{\frac{\alpha}{2}}$ is enough to guarantee any target rate in the absence of interference. Specifically, to guarantee an achievable rate of at least $R$ bps in the absence of interference, one should choose $P_{0}=\frac{N_{0}}{G}\left(2^{R}-1\right)$. In practice, almost all of the players will find a destination among their nearest neighbors at a distance that scales like $\frac{1}{\sqrt{N}}$ rather than $\sqrt{\frac{\log N}{N}}$. Hence, such a player can use power control and decrease his energy consumption by using only $P_{n}=\frac{P_{0}}{N^{\frac{\alpha}{2}}}$ and still obtain the required achievable rate.

Finally, we allow each player to be a destination to a maximum of $S>0$ players, which is fixed with respect to $N$. This assumption does not actually stand on its own. Rather it is naturally obtained from the requirement that the next hop of each player is one of his nearest neighbors since their number, on average, is constant with respect to $N$.

Our model is a building block for many communication scenarios. For instance, for duplex communication one can use parallel interference games, one for each direction, where in the second game the transmitter and receiver rules of each pair are reversed.

A toy example of our network is depicted in Figure 1 The destination of each player (dot) is shown with an arrow, and the type of the arrow (dashed, solid or gray) represents one of three available channels. Wired players are connected to the doted outgoing lines.

An alternative limit to the one taken in this work is to let the area of the region $\mathcal{D}$ grow linearly with $N$. This way the number of channels $K$ could be kept constant as $N \rightarrow \infty$ without rendering the average throughput to vanish due to the interference. In this limit the expected number of players in each channel goes to infinity and the mean-field approach, or concentration results such as [45], can be applied to the interference.

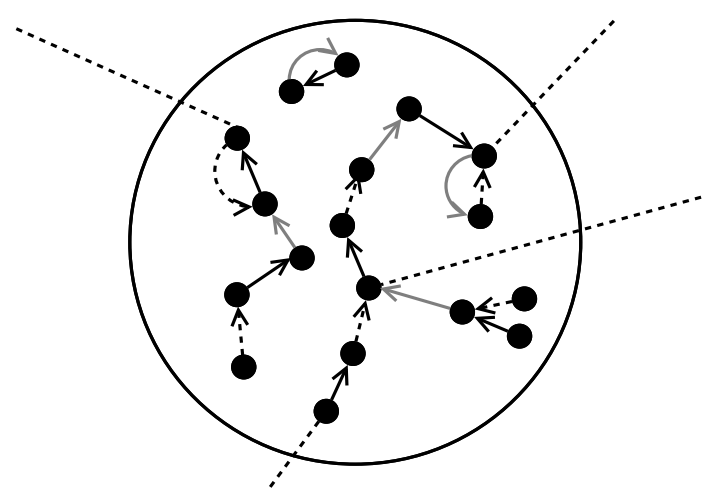

Figure 1: Illustration of the network for $N=18$ and $K=3$ 


\section{APPROXIMATE ASYNCHRONOUS BR DYNAMICS}

In this section we define our approximate BR dynamics, which we show are converging for asymptotically almost all interference games. The idea behind our convergence result is that although our game is not a congestion (or potential) game, it is a property of the typical realization game rather than of the distribution of the random game. While it is possible that some player may choose an action that decreases the sum-rate, he has no statistical incentive to do so. A player simply does not have enough information to choose an action that benefits other players. This implies that if each player's action is random enough, the average change in the sum-rate will be positive. Broadly speaking, this means that the average game can be thought of as a congestion game. Nevertheless, our dynamics should converge in almost all realizations of networks and the fact that the average one behaves well does not imply that most of them are.

Each realization possesses a large number of random variables: these are the channel gains of the large number of players $N$. Therefore, the statistics of the average action are in some sense similar to those of any player in the average game, and all that is left is that some mechanism will average over the actions and players. Fortunately, this type of mechanism is very natural in reality. We suggest the Approximate Asynchronous BR Dynamics to be this mechanism, as presented in this section. The randomness of these dynamics has two sources: the random acting player in each step, and the possibly random action that he chooses.

Our time axis is discrete, with index $t$, and in each step some random player chooses his action and the strategy profile changes. We want $\left\{\mathbf{a}_{\mathbf{t}}\right\}$ to converge to some epsilon pure Nash equilibrium.

Definition 5. A strategy profile $\left(a_{n}^{*}, \mathbf{a}_{-n}^{*}\right) \in A_{1} \times \ldots \times A_{N}$ is called an epsilon pure Nash equilibrium $(\varepsilon-\mathrm{PNE})$ if $u_{n}\left(a_{n}^{*}, \mathbf{a}_{-n}^{*}\right) \geq$ $u_{n}\left(a_{n} \mathbf{a}_{-n}^{*}\right)-\varepsilon$ for all $a_{n} \in A_{n}$ and all $n \in \mathcal{N}$.

If every player acts asynchronously, the acting player in a given step will emerge at random from some set of possible acting players. This can be implemented by random channel access schemes, similar in spirit to carrier-sense multiple access (CSMA) or the ALOHA protocol. We emphasize that the transmission itself needs not be of short duration as in the traditional schemes. In our dynamics, each player is using his current chosen channel constantly, until an opportunity to switch a channel arises.

Definition 6 (Non-Degenerate Schedule). Denote by $\mathcal{N}_{t}$ the set of players that can act on turn $t$ and their number by $N_{t}$. In an asynchronous dynamics with the schedule $\left\{\mathcal{N}_{t}\right\}$, the acting player on turn $t$ is chosen uniformly at random from $\mathcal{N}_{t}$. We say that the schedule $\left\{\mathcal{N}_{t}\right\}$ is non-degenerate, with respect to the decision rules $h_{n}: A_{1} \times \ldots \times A_{N} \rightarrow A_{1} \times \ldots \times A_{N}$, if for each $t \geq 0$ either it contains at least one player $n$ such that $h_{n}\left(\mathbf{a}_{t}\right) \neq \mathbf{a}_{t}$ or that $h_{n}\left(\mathbf{a}_{t}\right)=\mathbf{a}_{t}$ for each $n \in \mathcal{N}$.

In a degenerate schedule, there could be turns where only players who do not want to deviate have a chance to act. In a non-degenerate schedule this can only happen in an equilibrium. A degenerate schedule may lead to an infinite number of turns where nothing happens, which makes the question of convergence not well defined. Natural choices for a non-degenerate $\mathcal{N}_{t}$ are $\mathcal{N}_{t}=\mathcal{N}$ (all players) and $\mathcal{N}_{t}=\left\{n \mid B R_{\varepsilon, n}\left(\mathbf{a}_{t}\right) \neq \mathbf{a}_{t}\right\}$ (the deviating players). For these choices of $\mathcal{N}_{t}$ each player acts independently, and no centralized coordination is required. Many other interesting choices can be based on fully distributed 
protocols.

Now we define our dynamics, which are to choose at random from the set of $\frac{\varepsilon}{2}$-best actions, or keep the previous action if it is currently $\varepsilon$-best. The randomness of the action chosen by the acting player is a result of the indifference between the approximately best actions. Our non-degeneracy of $\left\{\mathcal{N}_{t}\right\}$ is with respect to these dynamics.

Definition 7. Let $\varepsilon>0$. The set of $\varepsilon$-best actions for player $n$ is defined as

$$
B_{\varepsilon}\left(\mathbf{a}_{-n}\right)=\left\{a \mid a \in A_{n}, u_{n}\left(a, \mathbf{a}_{-n}\right)+\varepsilon \geq \max _{a^{\prime} \in A_{n}} u_{n}\left(a^{\prime}, \mathbf{a}_{-n}\right)\right\}
$$

This is a finite and discrete set of channels. The $\varepsilon-\mathrm{BR}$ response function of player $n, B R_{\varepsilon, n}(\mathbf{a}, \omega): \omega \times A_{1} \times \ldots \times A_{N} \rightarrow$ $A_{1} \times \ldots \times A_{N}$, is defined as

$$
B R_{\varepsilon, n}(\mathbf{a}, \omega)=\left\{\begin{array}{cc}
\mathbf{a} & a_{n} \in B_{\varepsilon}\left(\mathbf{a}_{-n}\right) \\
\left(a_{\text {rand }, n}(\omega), \mathbf{a}_{-n}\right) & \text { o.w. }
\end{array}\right.
$$

where $a_{\text {rand }, n}(\omega): B_{\frac{\varepsilon}{2}}\left(\mathbf{a}_{-n}\right) \rightarrow B_{\frac{\varepsilon}{2}}\left(\mathbf{a}_{-n}\right)$ is a random variable which is uniformly distributed over $B_{\frac{\varepsilon}{2}}\left(\mathbf{a}_{-n}\right)$. For simplicity, through the paper we use the notation $B R_{\varepsilon, n}(\mathbf{a})$ instead, which is a random variable with distribution that is determined by a.

In order to prove convergence, we have to find some function of $\mathbf{a}_{\mathbf{t}}$, denoted by $X\left(\mathbf{a}_{\mathbf{t}}\right)$, for which the expectation over the random actions grows with each step. This will make $\left\{X\left(\mathbf{a}_{\mathbf{t}}\right), \mathbf{a}_{\mathbf{t}}\right\}$ a submartingale (i.e., a submartingale with respect to the sigma algebra generated by the sequence $\left\{\mathbf{a}_{\mathbf{t}}\right\}$ ). The formalization of our proof is based on the martingale convergence theorem [46, Page 89, Theorem 5.14]. Let $X\left(\mathbf{a}_{t}\right)$ be the sum of the achievable rates experienced by all players in their chosen channel, i.e.,

$$
X\left(\mathbf{a}_{t}\right)=\sum_{n=1}^{N} R_{n}\left(\mathbf{a}_{\mathbf{t}}\right)
$$

This is a real non-negative bounded function of the strategy profile. Recall that this is just the social welfare of an interference game. This means that this convergent function is aligned with our centralized objective and will guarantee that the resulting NE exhibits good performance. To prove convergence we have to show that $E\left\{X\left(\mathbf{a}_{\mathbf{t}+\mathbf{1}}\right)-X\left(\mathbf{a}_{\mathbf{t}}\right) \mid \mathbf{a}_{\mathbf{t}}\right\}$ is a non-negative function of $\mathbf{a}_{\mathbf{t}}$ for all $t \geq 0$, where the expectation is taken over the random acting player and the random action that he may choose. Let $\mathbf{a}_{\mathbf{t}+\mathbf{1}}=B R_{\varepsilon, n}\left(\mathbf{a}_{\mathbf{t}}\right)$, so player $n$ is the acting player at time $t$ and observe that

$$
X\left(\mathbf{a}_{\mathbf{t}+\mathbf{1}}\right)-X\left(\mathbf{a}_{\mathbf{t}}\right)=\Delta_{1}\left(\mathbf{a}_{\mathbf{t}}\right)+\Delta_{2}\left(\mathbf{a}_{\mathbf{t}}\right)+\Delta_{3}\left(\mathbf{a}_{\mathbf{t}}\right)
$$

where

$$
\Delta_{1}\left(\mathbf{a}_{\mathbf{t}}\right)=R_{n}\left(\mathbf{a}_{\mathbf{t}+\mathbf{1}}\right)-R_{n}\left(\mathbf{a}_{\mathbf{t}}\right)
$$

is the rate difference of the acting player,

$$
\Delta_{2}\left(\mathbf{a}_{\mathbf{t}}\right)=\sum_{\left\{m \mid a_{n, t+1}=a_{m, t}\right\}}\left(R_{m}\left(\mathbf{a}_{\mathbf{t}+\mathbf{1}}\right)-R_{m}\left(\mathbf{a}_{\mathbf{t}}\right)\right)
$$


is the rate difference caused by the additional interference to players in the channel that player $n$ is moving to, and

$$
\Delta_{3}\left(\mathbf{a}_{\mathbf{t}}\right)=\sum_{\left\{m \mid a_{n, t}=a_{m, t}\right\}}\left(R_{m}\left(\mathbf{a}_{\mathbf{t}+\mathbf{1}}\right)-R_{m}\left(\mathbf{a}_{\mathbf{t}}\right)\right)
$$

is the rate difference caused by the reduced interference to players in the channel that player $n$ is moving from.

All three are random variables with a distribution that depends on $\mathbf{a}_{\mathbf{t}}$. Specifically, if player $n$ is the acting player at time $t$ and $B R_{\varepsilon, n}\left(\mathbf{a}_{\mathbf{t}}\right)=\mathbf{a}_{\mathbf{t}}$ then $\Delta_{1}\left(\mathbf{a}_{\mathbf{t}}\right)=\Delta_{2}\left(\mathbf{a}_{\mathbf{t}}\right)=\Delta_{3}\left(\mathbf{a}_{\mathbf{t}}\right)=0$.

Our main result is the convergence of the approximate BR dynamics to an $\varepsilon$-PNE such that each $\varepsilon$-PNE is asymptotically $\varepsilon$-optimal in the max-min sense, and as well in the mean-rate. This result is stated as follows

Theorem 1 (Main Result). Assume that the players' locations are independently and uniformly randomly generated in some closed set $\mathcal{D} \subseteq \mathbb{R}^{2}$ with area $|\mathcal{D}|=\frac{1}{\lambda}$ for some $\lambda>0$. Also assume that the schedule $\left\{\mathcal{N}_{t}\right\}$ is non-degenerate. If $P_{\max }=$

$P_{0}\left(\frac{\log N}{N}\right)^{\frac{\alpha}{2}}$ for some $P_{0}>0$ and $\frac{N}{K}=l_{N} \geq 1$ satisfies $l_{N}=o\left(\left(\frac{N}{\log N}\right)^{\frac{\alpha}{\alpha+2}}\right)$ then, for any $\varepsilon>0$, the approximate BR dynamics converge to an $\varepsilon-P N E$ in a finite time with a probability that approaches 1 as $N \rightarrow \infty$. Denote by $\mathcal{P}_{\varepsilon}$ the random set of $\varepsilon-P N E$ of the realization game. For every $\delta>0$

$$
\operatorname{Pr}\left(\max _{\mathbf{a}^{*} \in \mathcal{P}_{\varepsilon}, n \in \mathcal{N}}\left|u_{n}\left(\mathbf{a}^{*}\right)-\log _{2}\left(1+\frac{g_{n, d(n)} P_{n}}{N_{0}}\right)\right| \leq \varepsilon+\delta\right) \rightarrow 1
$$

as $N \rightarrow \infty$.

The proof of Theorem 1 is delayed for the end of Section IV. According to this theorem, the $\varepsilon$-PNE of asymptotically almost all the interference games exhibit vanishing interference for each player. This fact on its own should not be surprising. There are $l_{N} \geq 1$ players on average in each channel. If $l_{N}$ is not growing too fast, or for simplicity is just a constant, it should be possible to design an allocation where all these sharing players are geometrically separated enough in $\mathcal{D}$. Due to the decreasing transmission power required for each player to communicate with his destination, this geometrical separation guarantees negligible interference for large enough $N$. A small enough interference is a desired property of any well-functioning network. The essence of our result is in the fact that approximate BR, that constitute a very simple and distributed algorithm, naturally tend to converge to this desired outcome. In other words, the dynamics of the interference game in this scenario can be thought of as a distributed and adaptive implementation of frequency band allocation over space (see [47]).

\section{Submartingality of the Sum-Rate Process}

We begin this section by proving that the process $X\left(\mathbf{a}_{\mathbf{t}}\right)=\sum_{n=1}^{N} R\left(I_{n}\left(\mathbf{a}_{t}\right)\right)$ is indeed a submartingale. More exactly, the sum-rate process is a submartingale for asymptotically almost all the interference games, so we want to show that

$$
\lim _{N \rightarrow \infty} \operatorname{Pr}\left(\forall t, E\left\{X\left(\mathbf{a}_{\mathbf{t}+\mathbf{1}}\right)-X\left(\mathbf{a}_{\mathbf{t}}\right) \mid \mathbf{a}_{\mathbf{t}}\right\} \geq 0\right)=1
$$

Note that the above probability is with respect to the channel gains that are generated at random at $t=0$, based on the locations of the players, and remain constant for all $t \geq 0$. The expectation is over the randomness of the acting player and his 
actions. This probability is our measure to "count the number" of interference games where convergence of the approximate BR occurs (how large the set of converging interference games is).

The interference game characteristics are determined by the geometry of its players. For instance, player $n$ 's transmitter strongly interferes players with a nearby destination, and player $n$ 's destination undergoes weak interference from far away transmitters. This is depicted in Figure 2. We want the first set of near players to be as small as possible while the second set of far players to be as large as possible. We define these sets as follows

Definition 8. Assume the players are located in some closed set $\mathcal{D} \subseteq \mathbb{R}^{2}$ with area $|\mathcal{D}|=\frac{1}{\lambda}$ for some $\lambda>0$. Define the set of near players, with respect to player $n$ 's transmitter, as

$$
\mathcal{N}_{\text {near }, n}=\left\{m\left|m \in \mathcal{N}, r_{n, d(m)} \leq \frac{1}{\sqrt{\pi \lambda}}\left(\frac{\log N}{N}\right)^{\frac{\alpha}{2 \alpha+4}},\right| \theta_{n, d(m)} \mid \leq \frac{\theta_{T}}{2}, d(m) \neq n\right\}
$$

and the set of far players, with respect to player $n$ 's receiver, for some $q>1$, as

$$
\mathcal{N}_{\text {far }, n}=\mathcal{N} \backslash\left\{m\left|m \in \mathcal{N}, r_{m, d(n)} \leq \sqrt{\frac{1}{2 \theta_{R} \lambda} \frac{q-1}{q l_{N}-1}},\right| \theta_{d(n), m} \mid \leq \frac{\theta_{R}}{2}\right\}
$$

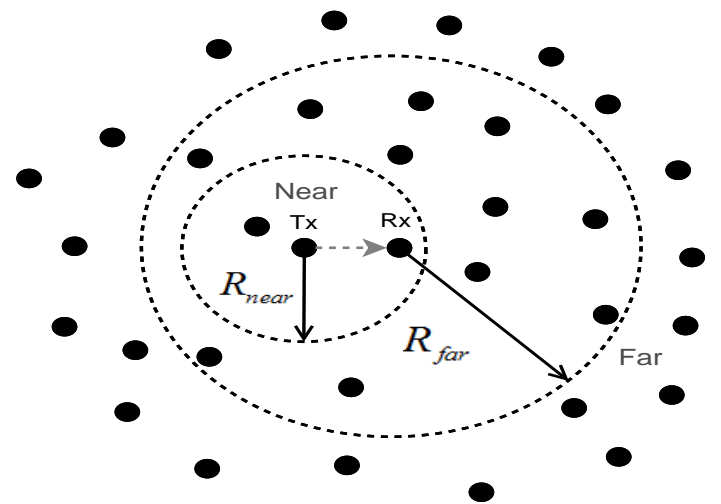

Figure 2: Illustration of the near and far regions.

The next lemma provides asymptotic probabilistic bounds for the cardinality of these two sets.

Lemma 1. Let $\frac{N}{K}=l_{N} \geq 1$ satisfy $l_{N}=o\left(\left(\frac{N}{\log N}\right)^{\frac{\alpha}{\alpha+2}}\right)$. Assume that the players' locations are independently and uniformly randomly generated in some closed set $\mathcal{D} \subseteq \mathbb{R}^{2}$ with area $|\mathcal{D}|=\frac{1}{\lambda}$ for some $\lambda>0$. Denote $N_{\text {near }, n}=\left|\mathcal{N}_{\text {near }, n}\right|$ and $N_{\text {far }, n}=\left|\mathcal{N}_{\text {far }, n}\right|$. Then

$$
\lim _{N \rightarrow \infty} \operatorname{Pr}\left(\max _{n \in \mathcal{N}} N_{\text {near }, n} \leq \frac{\theta_{T}}{\pi}\left(N^{2} \log ^{\alpha} N\right)^{\frac{1}{\alpha+2}}\right)=1
$$

and for any $q>1$

$$
\lim _{N \rightarrow \infty} \operatorname{Pr}\left(\min _{n \in \mathcal{N}} N_{f a r, n} \geq\left(1-\frac{1}{2} \frac{q-1}{q l_{N}-1}\right) N\right)=1
$$

where $\theta_{T}$ is the transmission beamforming angle (see Definition (4).

Proof: Denote the sector originated at player $n$ with angle $\theta$ (around an arbitrary line) and radius $t$ by $\Phi_{t, \theta, n}$, and the number of players in it (not including player $n$ ) by $N_{t, \theta, n}$. Due to the players' locations being uniformly and independently 
distributed, for each $n$ and $m$ we have

$$
\operatorname{Pr}\left(m \in \Phi_{t, \theta, n}\right)=\operatorname{Pr}\left(r_{n, m} \leq t \cdot I_{d}\left\{\left|\theta_{n, m}\right| \leq \frac{\theta}{2}\right\}\right) \underset{(a)}{\leq} \frac{\left|\Phi_{t, \theta, n}\right|}{|\mathcal{D}|} \underset{(b)}{=} \frac{\lambda \theta t^{2}}{2}
$$

where (a) follows from the possibility that part of $\Phi_{t, \theta, n}$, which has area $\frac{\theta t^{2}}{2}$, may fall outside $\mathcal{D}$ and (b) from $|\mathcal{D}|=\frac{1}{\lambda}$. From (15) we conclude that the random variable $N_{t, \theta, n}$ is stochastically dominated by a binomially random variable with $N$ trials and success probability $p=\frac{\lambda \theta t^{2}}{2}$, denoted $Y_{p}$. This is due to the fact there are actually only $N-1$ trials, and by (15), the success probability is smaller than $p$. Using this fact we get from Theorem A.1.12 in [48] that for all $N>0$ and $\beta>1$

$$
\operatorname{Pr}\left(N_{t, \theta, n} \geq \beta p N\right) \leq \operatorname{Pr}\left(Y_{p} \geq \beta p N\right) \leq\left(e^{\beta-1} \beta^{-\beta}\right)^{p N}
$$

To prove (13), observe that $N_{\text {near }, n}$ is distributed like $N_{t, \theta, n}$ for $t=\frac{1}{\sqrt{\pi \lambda}}\left(\frac{\log N}{N}\right)^{\frac{\alpha}{2 \alpha+4}}$ and $\theta=\theta_{T}$, so $p=\frac{\theta_{T}}{2 \pi}\left(\frac{\log N}{N}\right)^{\frac{\alpha}{\alpha+2}}$. By substituting $n=\frac{\theta_{T}}{\pi}\left(N^{2} \log ^{\alpha} N\right)^{\frac{1}{\alpha+2}}=2 p N$ in (16) we obtain for all $N>0$

$$
\operatorname{Pr}\left(N_{\text {near }, 1} \geq \frac{\theta_{T}}{\pi}\left(N^{2} \log ^{\alpha} N\right)^{\frac{1}{\alpha+2}}\right) \leq\left(e \cdot 2^{-2}\right)^{\frac{\theta_{T}}{2 \pi}\left(N^{2} \log ^{\alpha} N\right)^{\frac{1}{\alpha+2}}} .
$$

We conclude by the union bound that

$$
\begin{aligned}
\operatorname{Pr}\left(\max _{n \in \mathcal{N}} N_{\text {near }, n} \geq \frac{\theta_{T}}{\pi}\left(N^{2} \log ^{\alpha} N\right)^{\frac{1}{\alpha+2}}\right)= & \operatorname{Pr}\left(\bigcup_{n=1}^{N}\left\{N_{\text {near }, n} \geq \frac{\theta_{T}}{\pi}\left(N^{2} \log ^{\alpha} N\right)^{\frac{1}{\alpha+2}}\right\}\right) \leq \\
& N \operatorname{Pr}\left(N_{\text {near }, 1} \geq \frac{\theta_{T}}{\pi}\left(N^{2} \log ^{\alpha} N\right)^{\frac{1}{\alpha+2}}\right) \leq N\left(e \cdot 2^{-2}\right)^{\frac{\theta_{T}}{2 \pi}\left(N^{2} \log ^{\alpha} N\right)^{\frac{1}{\alpha+2}}}
\end{aligned}
$$

so $\lim _{N \rightarrow \infty} \operatorname{Pr}\left(\max _{n \in \mathcal{N}} N_{\text {near }, n} \leq \frac{\theta_{T}}{\pi}\left(N^{2} \log ^{\alpha} N\right)^{\frac{1}{\alpha+2}}\right)=1$.

To prove (14), observe that we can write $N_{\text {far }, n}=(N-1)-\tilde{N}_{n}$, where $\tilde{N}_{n}$ is distributed like $N_{t, \theta, d(n)}$ for $t=\sqrt{\frac{1}{2 \theta_{R} \lambda} \frac{q-1}{q l_{N}-1}}$ and $\theta=\theta_{R}$, so $p=\frac{1}{4} \frac{q-1}{q l_{N}-1}$. By substituting $n=\frac{1}{2} \frac{q-1}{q l_{N}-1} N-1=\left(2-\frac{4}{N} \frac{q l_{N}-1}{q-1}\right) p N$ in (16) and denoting $\eta=\frac{4}{N} \frac{q l_{N}-1}{q-1}$ we obtain that for all $N>0$

$$
\operatorname{Pr}\left(\tilde{N}_{1} \geq \frac{1}{2} \frac{q-1}{q l_{N}-1} N-1\right) \leq\left(e^{1-\eta} \cdot(2-\eta)^{-(2-\eta)}\right)^{\frac{1}{4} \frac{q-1}{q l_{N}-1} N}
$$

We conclude by the union bound that

$$
\begin{aligned}
\operatorname{Pr}\left(\min _{n \in \mathcal{N}} N_{\text {far }, n} \leq\left(1-\frac{1}{2} \frac{q-1}{q l_{N}-1}\right) N\right)= & \operatorname{Pr}\left(\bigcup_{n=1}^{N}\left\{N-N_{\text {far }, n} \geq \frac{1}{2} \frac{q-1}{q l_{N}-1} N\right\}\right) \leq \\
& N \operatorname{Pr}\left(\tilde{N}_{1} \geq \frac{1}{2} \frac{q-1}{q l_{N}-1} N-1\right) \leq N\left(e^{1-\eta} \cdot(2-\eta)^{-(2-\eta)}\right)^{\frac{1}{4} \frac{q-1}{q l_{N}-1} N}
\end{aligned}
$$

so $\lim _{N \rightarrow \infty} \operatorname{Pr}\left(\min _{n \in \mathcal{N}} N_{\text {far }, n} \geq\left(1-\frac{1}{2} \frac{q-1}{q l_{N}-1}\right) N\right)=1$ (since $\eta \rightarrow 0$ as $N \rightarrow \infty$ ).

The following simple lemma will be useful for some of our calculations.

Lemma 2. For any $a, b, \triangle I>0$,

$$
\log _{2}\left(1+\frac{a}{b}\right)-\log _{2}\left(1+\frac{a}{b+\triangle I}\right) \leq \frac{a}{a+b} \frac{\triangle I}{b \ln (2)}
$$


Proof: This follows by

$$
\begin{array}{r}
\log _{2}\left(1+\frac{a}{b}\right)-\log _{2}\left(1+\frac{a}{b+\triangle I}\right)=\log _{2}\left(1+\frac{\frac{a}{b}-\frac{a}{b+\triangle I}}{1+\frac{a}{b+\triangle I}}\right)=\log _{2}\left(1+\frac{a}{b} \frac{\triangle I}{a+\triangle I+b}\right) \leq \\
\log _{2}\left(1+\frac{a}{a+b} \frac{\triangle I}{b}\right) \leq \frac{a}{(a)} \frac{\Delta+b}{a \ln (2)}
\end{array}
$$

where (a) is due to $\log _{2}(1+x) \leq \frac{x}{\ln (2)}$ for all $x \geq 0$.

Now we turn to exploit the above results for our purpose of showing the submartingality of the sum-rate process. The following lemma shows that the set $B_{\varepsilon}\left(\mathbf{a}_{-n}\right)$ is asymptotically large, and each action in it is asymptotically approximately optimal (interference free). This supplies the necessary averaging effect over the random choice of an action among the approximately best actions.

Lemma 3. Assume that the players' locations are independently and uniformly randomly generated in some closed set $\mathcal{D} \subseteq \mathbb{R}^{2}$ with area $|\mathcal{D}|=\frac{1}{\lambda}$ for some $\lambda>0$. If $P_{\max }=P_{0}\left(\frac{\log N}{N}\right)^{\frac{\alpha}{2}}$ for some $P_{0}>0$ and $\frac{N}{K}=l_{N} \geq 1$ satisfies $l_{N}=o\left(\left(\frac{N}{\log N}\right)^{\frac{\alpha}{\alpha+2}}\right)$, then for any $q>1$ and $\varepsilon>0$

$$
\lim _{N \rightarrow \infty} \operatorname{Pr}\left(\forall \mathbf{a}, \min _{n \in \mathcal{N}^{N}}\left|B_{\varepsilon}\left(\mathbf{a}_{-n}\right)\right| \geq \frac{1}{2 l_{N}}\left(1-\frac{1}{q}\right) N\right)=1
$$

Furthermore,

$$
\max _{n \in \mathcal{N} \mathbf{a} \in A_{1} \times \ldots \times A_{N}}\left|u_{n}\left(B R_{n}(\mathbf{a})\right)-\log _{2}\left(1+\frac{g_{n, d(n)} P_{n}}{N_{0}}\right)\right| \rightarrow 0
$$

in probability as $N \rightarrow \infty$.

Proof: See Appendix A.

No doubt that a deviating player can only improve his utility, but the following lemma shows that on average over his chosen action, he also causes asymptotically negligible interference to other players. This establishes that the sum-rate process is indeed a submartingale.

Lemma 4. Assume that the players' locations are independently and uniformly randomly generated in some closed set $\mathcal{D} \subseteq \mathbb{R}^{2}$ with area $|\mathcal{D}|=\frac{1}{\lambda}$ for some $\lambda>0$. Also assume that $P_{\max }=P_{0}\left(\frac{\log N}{N}\right)^{\frac{\alpha}{2}}$ for some $P_{0}>0$ and that $\frac{N}{K}=l_{N} \geq 1$ satisfies $l_{N}=o\left(\left(\frac{N}{\log N}\right)^{\frac{\alpha}{\alpha+2}}\right)$. Let $\varepsilon>0$ and the acting player at time $t$ be player $n$. Denote by $E_{n}$ the expectation over his chosen channel. Then the probability that for every $\mathbf{a}_{t}$ and $n$ such that $\mathbf{a}_{\mathbf{t}+\mathbf{1}}=B R_{\varepsilon, n}\left(\mathbf{a}_{t}\right) \neq \mathbf{a}_{t}$, for any $t \geq 0$

$$
E_{n}\left\{\Delta_{1}\left(\mathbf{a}_{\mathbf{t}}\right)+\Delta_{2}\left(\mathbf{a}_{\mathbf{t}}\right) \mid \mathbf{a}_{\mathbf{t}}\right\}>0
$$

approaches 1 a $N \rightarrow \infty$, where the probability is with respect to the random interference game, and $\Delta_{1}\left(\mathbf{a}_{\mathbf{t}}\right), \Delta_{2}\left(\mathbf{a}_{\mathbf{t}}\right)$ are defined in (7), (8).

\section{Proof: See Appendix B.}

We conclude this section by proving our main result.

Proof of Theorem 1: Let $\varepsilon>0$. According to Lemma 4 (see (53) the probability that for every $\mathbf{a}_{t}$ and $n$ such that 
$\mathbf{a}_{\mathbf{t}+\mathbf{1}}=B R_{\varepsilon, n}\left(\mathbf{a}_{\mathbf{t}}\right) \neq \mathbf{a}_{t}$, the following holds for all $t \geq 0$

$$
E_{n}\left\{\Delta_{1}\left(\mathbf{a}_{\mathbf{t}}\right)+\Delta_{2}\left(\mathbf{a}_{\mathbf{t}}\right)+\Delta_{3}\left(\mathbf{a}_{\mathbf{t}}\right) \mid \mathbf{a}_{\mathbf{t}}\right\} \geq \frac{\varepsilon}{4}
$$

approaches 1 as $N \rightarrow \infty$, because $\Delta_{3}\left(\mathbf{a}_{\mathbf{t}}\right) \geq 0$ for each $\mathbf{a}_{t}$. Clearly $X\left(\mathbf{a}_{\mathbf{t}+\mathbf{1}}\right)=X\left(\mathbf{a}_{\mathbf{t}}\right)$ if $\mathbf{a}_{\mathbf{t}}$ is an $\varepsilon$-PNE. Summing over all $n \in \mathcal{N}_{t}$ and dividing by $\frac{1}{N_{t}}$ we obtain that

$$
\lim _{N \rightarrow \infty} \operatorname{Pr}\left(\forall t, E\left\{X\left(\mathbf{a}_{\mathbf{t}+\mathbf{1}}\right)-X\left(\mathbf{a}_{\mathbf{t}}\right) \mid \mathbf{a}_{\mathbf{t}}\right\} \geq 0\right)=1
$$

Observe that the sum-rate process is bounded, since for each $t \geq 0$, with probability 1

$$
\left|X\left(\mathbf{a}_{\mathbf{t}}\right)\right| \leq \sum_{n=1}^{N} \log _{2}\left(1+\frac{g_{n, d(n)} P_{n}}{N_{0}}\right)
$$

If indeed (26) holds, then from the Martingale Convergence Theorem [46, Page 89, Theorem 5.14] we conclude that there exists a random variable $X$ such that $X\left(\mathbf{a}_{\mathbf{t}}\right) \rightarrow X$ almost surely and $E\{|X|\}<\infty$. Due to (28), the almost sure convergence also implies $L^{1}$ convergence (bounded random variables are trivially uniformly integrable), so for $\varepsilon^{\prime}=\frac{\varepsilon}{8 N^{2}}$ there exists an $T>0$ such that for each $t \geq T$

$$
E\left\{\left|X\left(\mathbf{a}_{t+1}\right)-X\left(\mathbf{a}_{t}\right)\right|\right\} \underset{(a)}{\leq} E\left\{\left|X\left(\mathbf{a}_{t+1}\right)-X\right|\right\}+E\left\{\left|X\left(\mathbf{a}_{t}\right)-X\right|\right\}<2 \varepsilon^{\prime}
$$

where (a) follows from the triangle inequality. Denote by $\mathcal{P}_{\varepsilon}$ the set of $\varepsilon$-PNE points of the game. We conclude that with probability (with respect to the random game) that approaches 1 as $N \rightarrow \infty$

$$
\begin{aligned}
& E\left\{X\left(\mathbf{a}_{\mathbf{T}+\mathbf{1}}\right)-X\left(\mathbf{a}_{\mathbf{T}}\right)\right\} \underset{(a)}{=} \sum_{\mathbf{a} \in A_{1} \times \ldots \times A_{n}} E\left\{X\left(\mathbf{a}_{\mathbf{T}+\mathbf{1}}\right)-X\left(\mathbf{a}_{\mathbf{T}}\right) \mid \mathbf{a}_{\mathbf{T}}=\mathbf{a}\right\} \operatorname{Pr}\left(\mathbf{a}_{\mathbf{T}}=\mathbf{a}\right)= \\
& \sum_{\mathbf{a} \in \mathcal{P}_{\varepsilon}} E\left\{X\left(\mathbf{a}_{\mathbf{T}+\mathbf{1}}\right)-X\left(\mathbf{a}_{\mathbf{T}}\right) \mid \mathbf{a}_{\mathbf{T}}=\mathbf{a}\right\} \operatorname{Pr}\left(\mathbf{a}_{\mathbf{T}}=\mathbf{a}\right)+\sum_{\mathbf{a} \notin \mathcal{P}_{\varepsilon}} E\left\{X\left(\mathbf{a}_{\mathbf{T}+\mathbf{1}}\right)-X\left(\mathbf{a}_{\mathbf{T}}\right) \mid \mathbf{a}_{\mathbf{T}}=\mathbf{a}\right\} \operatorname{Pr}\left(\mathbf{a}_{\mathbf{T}}=\mathbf{a}\right) \underset{(b)}{\geq} \\
& \frac{\varepsilon}{4 N_{t}} \sum_{\mathbf{a} \notin \mathcal{P}_{\varepsilon}} \operatorname{Pr}\left(\mathbf{a}_{\mathbf{T}}=\mathbf{a}\right) \geq \frac{\varepsilon}{4 N} \operatorname{Pr}\left(\mathbf{a}_{\mathbf{T}} \notin \mathcal{P}_{\varepsilon}\right)
\end{aligned}
$$

where (a) follows from the law of total expectation and (b) is from averaging (26) over $N_{t}$, relying on the non-degeneracy of the schedule $\left\{\mathcal{N}_{t}\right\}$. Inequality (b) is also due to $X\left(\mathbf{a}_{\mathbf{T}+\mathbf{1}}\right)=X\left(\mathbf{a}_{\mathbf{T}}\right)$ with probability 1 for all $\mathbf{a}_{\mathbf{T}} \in \mathcal{P}_{\varepsilon}$. Note that $\operatorname{Pr}\left(\mathbf{a}_{\mathbf{T}} \notin \mathcal{P}_{\varepsilon}\right)$ is with respect to the random player and his actions (and not the random game). From (30)

$$
\operatorname{Pr}\left(\mathbf{a}_{\mathbf{T}} \notin \mathcal{P}_{\varepsilon}\right) \leq \frac{4 N}{\varepsilon} E\left\{X\left(\mathbf{a}_{\mathbf{T}+\mathbf{1}}\right)-X\left(\mathbf{a}_{\mathbf{T}}\right)\right\} \leq \frac{4 N}{\varepsilon} E\left\{\left|X\left(\mathbf{a}_{T+1}\right)-X\left(\mathbf{a}_{T}\right)\right|\right\} \underset{(a)}{<} \frac{1}{N}
$$

where (a) is from (29), and hence $\operatorname{Pr}\left(\mathbf{a}_{\mathbf{T}} \notin \mathcal{P}_{\varepsilon}\right) \rightarrow 0$ as $N \rightarrow \infty$.

Part 2 follows by the definition of an $\varepsilon$-PNE, since for each $n$ and each $\mathbf{a}^{*} \in \mathcal{P}_{\varepsilon}$

$$
u_{n}\left(\mathbf{a}^{*}\right) \geq u_{n}\left(B R_{n}\left(\mathbf{a}^{*}\right)\right)-\varepsilon
$$


combining this with (24) from Lemma 3, we obtain (10). Finally, if $\max _{n \in \mathcal{N}}\left|u_{n}\left(\mathbf{a}^{*}\right)-\log _{2}\left(1+\frac{g_{n, d(n)} P_{n}}{N_{0}}\right)\right| \leq \varepsilon+\delta$ then also

$$
\frac{1}{N} \sum_{n=1}^{N} u_{n}\left(\mathbf{a}^{*}\right) \geq \frac{1}{N} \sum_{n=1}^{N} \log _{2}\left(1+\frac{g_{n, d(n)} P_{n}}{N_{0}}\right)-\varepsilon-\delta .
$$

It is worth mentioning that although the above theorem uses the non-degeneracy of the schedule $\left\{\mathcal{N}_{t}\right\}$, it is not necessary. Any schedule that ensures that each player gets an opportunity to act infinitely often is enough. This of course might be true even if on some turns all the possible acting players do not want to change their actions. Nevertheless, different schedules will result in different convergence times in terms of steps. The conversion between the number of steps until convergence to absolute time is dependent on the random access scheme.

\section{Convergence Time}

Our results from last section regarding the average change in the sum-rate in each step can be used to draw conclusions about the convergence time of the dynamics. In (approximate) BR dynamics, each player only considers the current strategy profile for his decision, and therefore these dynamics induce a Markov chain on $\left\{\mathbf{a}_{\mathbf{t}}\right\}$. From this perspective, our result from the last section establishes that this Markov chain is absorbing (from every state it is possible to reach an absorbing state), and hence converges to one of its absorbing states ( $\varepsilon$-PNE points). Note that this is equivalent to the definition of a weakly acyclic game. This Markovity is the key to the following lemma.

Lemma 5. Let $\left\{X\left(\mathbf{a}_{\mathbf{t}}\right)\right\}$ be the sum-rate process induced by the approximate BR dynamics and $k(\mathbf{a}): A_{1} \times \ldots \times A_{n} \rightarrow \mathbb{R}$. If for each a and each $t \geq 0$

$$
E\left\{X\left(\mathbf{a}_{\mathbf{t + 1}}\right)-X\left(\mathbf{a}_{\mathbf{t}}\right) \mid \mathbf{a}_{\mathbf{t}}=\mathbf{a}\right\} \geq k(\mathbf{a})
$$

then

$$
E\left\{X\left(\mathbf{a}_{\mathbf{T}}\right) \mid \mathbf{a}_{0}\right\} \geq X\left(\mathbf{a}_{0}\right)+E\left\{\sum_{t=0}^{T-1} k\left(\mathbf{a}_{t}\right) \mid \mathbf{a}_{0}\right\}
$$

Proof: We can iterate on (34) in the following manner

$$
\begin{gathered}
E\left\{X\left(\mathbf{a}_{\mathbf{t}+\mathbf{2}}\right)-X\left(\mathbf{a}_{\mathbf{t}}\right) \mid \mathbf{a}_{\mathbf{t}}, \ldots, \mathbf{a}_{0}\right\}=E\left\{X\left(\mathbf{a}_{\mathbf{t}+\mathbf{2}}\right)-X\left(\mathbf{a}_{\mathbf{t}+\mathbf{1}}\right) \mid \mathbf{a}_{\mathbf{t}}, \ldots, \mathbf{a}_{0}\right\}+E\left\{X\left(\mathbf{a}_{\mathbf{t}+\mathbf{1}}\right)-X\left(\mathbf{a}_{\mathbf{t}}\right) \mid \mathbf{a}_{\mathbf{t}}, \ldots, \mathbf{a}_{0}\right\} \underset{(a)}{=} \\
E\left\{E\left\{X\left(\mathbf{a}_{\mathbf{t}+\mathbf{2}}\right)-X\left(\mathbf{a}_{\mathbf{t}+\mathbf{1}}\right) \mid \mathbf{a}_{\mathbf{t}+\mathbf{1}}, \ldots, \mathbf{a}_{0}\right\} \mid \mathbf{a}_{\mathbf{t}}, \ldots, \mathbf{a}_{0}\right\}+E\left\{X\left(\mathbf{a}_{\mathbf{t}+\mathbf{1}}\right)-X\left(\mathbf{a}_{\mathbf{t}}\right) \mid \mathbf{a}_{\mathbf{t}}, \ldots, \mathbf{a}_{0}\right\} \underset{(b)}{=} \\
E\left\{E\left\{X\left(\mathbf{a}_{\mathbf{t}+\mathbf{2}}\right)-X\left(\mathbf{a}_{\mathbf{t}+\mathbf{1}}\right) \mid \mathbf{a}_{\mathbf{t}+\mathbf{1}}\right\} \mid \mathbf{a}_{\mathbf{t}}\right\}+E\left\{X\left(\mathbf{a}_{\mathbf{t}+\mathbf{1}}\right)-X\left(\mathbf{a}_{\mathbf{t}}\right) \mid \mathbf{a}_{\mathbf{t}}\right\} \underset{(c)}{\geq} E\left\{k\left(\mathbf{a}_{\mathbf{t}+\mathbf{1}}\right)+k\left(\mathbf{a}_{\mathbf{t}}\right) \mid \mathbf{a}_{\mathbf{t}}\right\}
\end{gathered}
$$

where (a) follows from the generalized law of total expectation, (b) from the Markovity of the process and (c) from (34). Inequality (35) follows by iterating $T$ times on (34) starting from $t=0$.

Using the above lemma we prove the next proposition.

Proposition 1. Assume that the players' locations are independently and uniformly randomly generated in some closed set $\mathcal{D} \subseteq \mathbb{R}^{2}$ with area $|\mathcal{D}|=\frac{1}{\lambda}$ for some $\lambda>0$. Also assume that $P_{\max }=P_{0}\left(\frac{\log N}{N}\right)^{\frac{\alpha}{2}}$ for some $P_{0}>0$ and that $\frac{N}{K}=$ 
$l_{N} \geq 1$ satisfies $l_{N}=o\left(\left(\frac{N}{\log N}\right)^{\frac{\alpha}{\alpha+2}}\right)$. Let $\varepsilon>0$. Denote by $\mathcal{P}_{\varepsilon}$ the set of $\varepsilon-P N E$ points of the game. Denote $\bar{C}=$ $\frac{1}{N} \sum_{n=1}^{N} \log _{2}\left(1+\frac{g_{n, d(n)} P_{n}}{N_{0}}\right)$. Define the convergence time of the approximate BR dynamics for an interference game by $T_{\text {con }}=\min _{t \geq 0}\left\{t \mid \mathbf{a}_{\mathbf{t}} \in \mathcal{P}_{\varepsilon}\right\}$. Assume $T_{\text {con }}<\infty$ (which happens with probability that approaches 1 as $N \rightarrow \infty$, with respect to the random game).

1) If $\left\{\mathcal{N}_{t}\right\}$ is non-degenerate then $\operatorname{Pr}\left(T_{\text {con }} \geq L \frac{4 \bar{C}}{\varepsilon} N^{2}\right) \leq \frac{1}{L}$ for each $L>0$.

2) If $\mathcal{N}_{t}=\left\{n \mid B R_{\varepsilon, n}\left(\mathbf{a}_{\mathbf{t}}\right) \neq \mathbf{a}_{t}\right\}$ for each $t \geq 0$ then $\operatorname{Pr}\left(T_{\text {con }} \geq L \frac{4 \bar{C}}{\varepsilon} N\right) \leq \frac{1}{L}$ for each $L>0$.

where the probabilities are with respect to the random players and actions.

Proof: Following the proof of Theorem 1 (see (26), we know that the probability that for every $\mathbf{a}_{t}$ and $n$ such that $\mathbf{a}_{\mathbf{t}+\mathbf{1}}=B R_{\varepsilon, n}\left(\mathbf{a}_{\mathbf{t}}\right) \neq \mathbf{a}_{t}$, the following holds for all $t \geq 0$

$$
E_{n}\left\{X\left(\mathbf{a}_{\mathbf{t}+\mathbf{1}}\right)-X\left(\mathbf{a}_{\mathbf{t}}\right) \mid \mathbf{a}_{\mathbf{t}}\right\} \geq \frac{\varepsilon}{4}
$$

approaches 1 as $N \rightarrow \infty$, where $E_{n}$ denotes the expectation over the random action of player $n$. Define the set of deviating players at time $t$ as $\mathcal{N}_{d, t}=\left\{n \mid n \in \mathcal{N}_{t}, B R_{\varepsilon, n}\left(\mathbf{a}_{t}\right) \neq \mathbf{a}_{t}\right\}$ and denote their number by $N_{d, t}$. From the non-degeneracy of $\left\{\mathcal{N}_{t}\right\}$ we know that $N_{d, t} \geq 1$ and hence, by averaging over $\mathcal{N}_{t}$ and using (37), we obtain for each $t<T_{\text {con }}$

$$
E\left\{X\left(\mathbf{a}_{\mathbf{t}+\mathbf{1}}\right)-X\left(\mathbf{a}_{\mathbf{t}}\right) \mid \mathbf{a}_{\mathbf{t}}\right\} \geq \frac{N_{d, t}}{4 N_{t}} \varepsilon
$$

Therefore by Lemma 5 we get, for each $\mathbf{a}_{0} \in A_{1} \times \ldots \times A_{n}$

$$
E\left\{X\left(\mathbf{a}_{T_{\text {con }}}\right) \mid \mathbf{a}_{0}\right\} \geq X\left(\mathbf{a}_{0}\right)+E\left\{\sum_{t=0}^{T_{\text {con }}-1} k\left(\mathbf{a}_{t}\right) \mid \mathbf{a}_{0}\right\} \underset{(a)}{\geq} \frac{\varepsilon}{4} E\left\{\sum_{t=0}^{T_{\text {con }}-1} \frac{N_{d, t}}{N_{t}} \mid \mathbf{a}_{0}\right\} \geq \frac{\varepsilon}{4} \min _{t} \frac{N_{d, t}}{N_{t}} E\left\{T_{\text {con }} \mid \mathbf{a}_{0}\right\}
$$

where (a) follows from $X\left(\mathbf{a}_{0}\right) \geq 0$ and $k\left(\mathbf{a}_{t}\right)=\frac{\varepsilon}{4} \frac{N_{d, t}}{N_{t}}$. Now define

$$
\hat{T}=\frac{4 N \bar{C}}{\varepsilon} \frac{1}{\min _{t} \frac{N_{d, t}}{N_{t}}}
$$

and observe that we must have $E\left\{T_{\text {con }} \mid \mathbf{a}_{0}\right\} \leq \hat{T}$ for each $\mathbf{a}_{0} \in A_{1} \times \ldots \times A_{n}$, otherwise by (39) we get $E\left\{X\left(\mathbf{a}_{\mathbf{T}_{\text {con }}}\right) \mid \mathbf{a}_{0}\right\}>$ $N \bar{C}$, which is a contradiction (see (28) $)$. By Markov inequality we get, for each $L>0$

$$
\operatorname{Pr}\left(T_{\text {con }} \geq L \hat{T}\right) \leq \frac{E\left\{T_{\text {con }}\right\}}{L \hat{T}} \leq \frac{1}{L}
$$

The two parts of this proposition follow from (40), because if $\left\{\mathcal{N}_{t}\right\}$ is non-degenerate then $\min _{t} \frac{N_{d, t}}{N_{t}} \geq \frac{1}{N}$, and if $\mathcal{N}_{t}=$ $\left\{n \mid B R_{\varepsilon, n}\left(\mathbf{a}_{t}\right) \neq \mathbf{a}_{t}\right\}$ for each $t \geq 0$ then $\min _{t} \frac{N_{d, t}}{N_{t}}=1$.

\section{Simulation Results}

We simulated $K$ channels and $N$ players independently and uniformly at random on a two-dimensional disk with radius $R_{d i s k}=10 \mathrm{~m}$. The channel gains were chosen according to (1) with wavelength $\nu=\frac{c}{2.4 \cdot 10^{9}}$ and $\alpha=3.5$. Each player's destination was drawn randomly from his five nearest neighbors. The transmission power of each player was chosen such that $S N R=20 d B$ in the absence of interference. These two features are essentially a power control mechanism that makes $P_{n}$ 
proportional to $\frac{1}{N^{\frac{\alpha}{2}}}$ on average over the random network, as it appears in our system model. In each iteration we chose a player at random from the set of players who want to switch a channel, i.e., $\mathcal{N}_{t}=\left\{n \mid B R_{\varepsilon, n}\left(\mathbf{a}_{\mathbf{t}}\right) \neq \mathbf{a}_{t}\right\}$. This player performed his approximate BR with some $\varepsilon$. Unless otherwise stated, the beamforming angles were $\theta_{T}=\theta_{R}=2 \pi$.

Figure 3 shows the convergence for a single realization with $N=50, K=5$ and $\varepsilon=0.1$. The beamforming angles were chosen to be $\theta_{T}=\frac{2 \pi}{3}$ and $\theta_{R}=2 \pi$. The martingale property of the mean-rate is clearly shown, as some of the iterations result in a mean-rate decrease. We can see that although $N$ is relatively small, the average mean-rate at equilibrium is not significantly smaller than $\log _{2}(1+100)=6.66$ and that the minimal rate converges to some reasonable value.

Figure 4 shows the empirical CDFs, based on 100 interference game realizations, of the convergence time for $N=$ $50,100,200,300,400, \varepsilon=0.1$ and $l_{N}=\frac{N}{K}=10$. It is evident that convergence to an $\varepsilon$-PNE indeed occurs, and it only requires a few actions on average from each player. The average convergence times are $78,156,249,353,443$, which are better than linear with $N$ as expected by Proposition 1

Figure 5 shows the mean rate as a function of the system load $l_{N}$, based on 100 interference game realizations, for $N=300$, $\varepsilon=0.5$ and $l_{N}=1,2,3,4,5,6,10,15,20$. We compare our results to those of a random allocation and a time-frequency multiple access allocation (TDMA/FDMA or orthogonal FDMA). Trivially, a TDMA/FDMA scheme achieves $R\left(l_{N}\right)=\frac{\log _{2}(101)}{l_{N}}$ for all players or $R\left(l_{N}\right)=\frac{\log _{2}\left(1+l_{N} \cdot 100\right)}{l_{N}}$ if the power limitation is over a frame. Our approximate BR outperforms all of these schemes for any $l_{N}$, and with an increasing ratio. For example, for $l_{N}=2$ our mean-rate is 1.7 times better than that of TDMA/FDMA and for $l_{N}=20$ the ratio is 6.9 .

Figure 6 presents the trade-off introduced by $\varepsilon$ between the convergence time and the rates. We used $N=200$ and $K=50$, for $\varepsilon=0.01,0.1,0.5,1,1.5,2,2.5$ and averaged the results over 200 realizations. Clearly, the convergence time decreases (improves) with $\varepsilon$ and so do the rates (get worse). For both the convergence time and the rates the effect of $\varepsilon$ seems to be slower than linear with a unit slope. This implies that the aforementioned trade-off is non-trivial. While the rate improvement of the acting player is always at least $\frac{\varepsilon}{2}$, in many of the turns (especially the initial ones) it is much larger and unaffected by $\varepsilon$. Regarding the rates, the mean rates are much less affected by $\varepsilon$ than the minimal rates. This is to be expected since the distribution of the rates of the channels in $B_{\varepsilon}\left(\mathbf{a}_{-n}\right)$ is far from being uniform between $R_{n}(0)-\varepsilon$ and $R_{n}(0)$. Most of the channels in $B_{\varepsilon}\left(\mathbf{a}_{-n}\right)$ result in a rate that is close to $R_{n}(0)$, with nothing to do with $\varepsilon$. This determines the average rate of the players. On the other hand, the worst channels in $B_{\varepsilon}\left(\mathbf{a}_{-n}\right)$, that result in the minimal rates, are (asymptotically) bounded from below by $R_{n}(0)-\varepsilon$, and were originally chosen by the corresponding players to be better than $R_{n}(0)-\frac{\varepsilon}{2}$. Indeed, we see that the minimal rates decrease with $\varepsilon$ with a slope between $\frac{1}{2}$ and 1 .

\section{CONCLUSION}

In this work, we proved the convergence of asynchronous approximate BR dynamics in a class of games called interference games, which model a natural conflict in many wireless network scenarios. We generated an interference game at random by generating the players' locations uniformly and independently at random at some area $\mathcal{D}$, and set the channel gains accordingly. Although interference games tend not to be potential games, we were able to show that the approximate BR dynamics converge to an approximate Nash equilibrium (NE) for almost all of the interference games, asymptotically in the number of players. The 


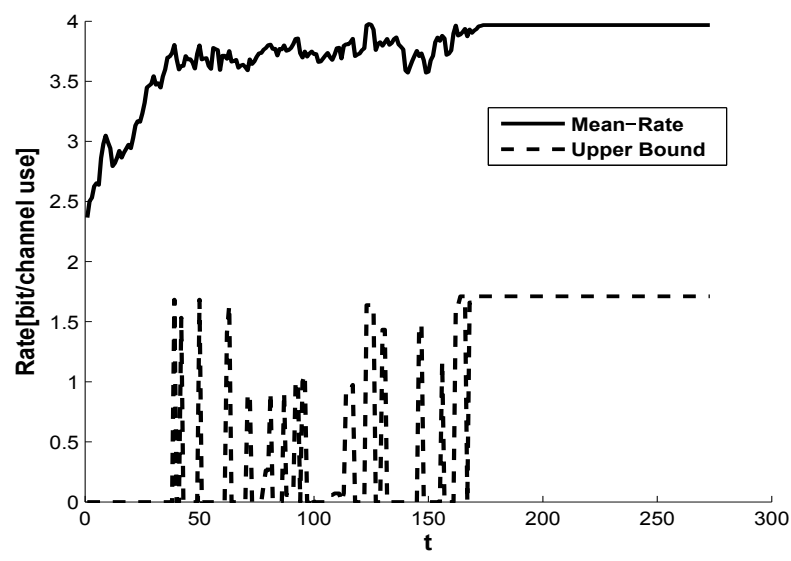

Figure 3: The mean and minimal rate of a single realization.

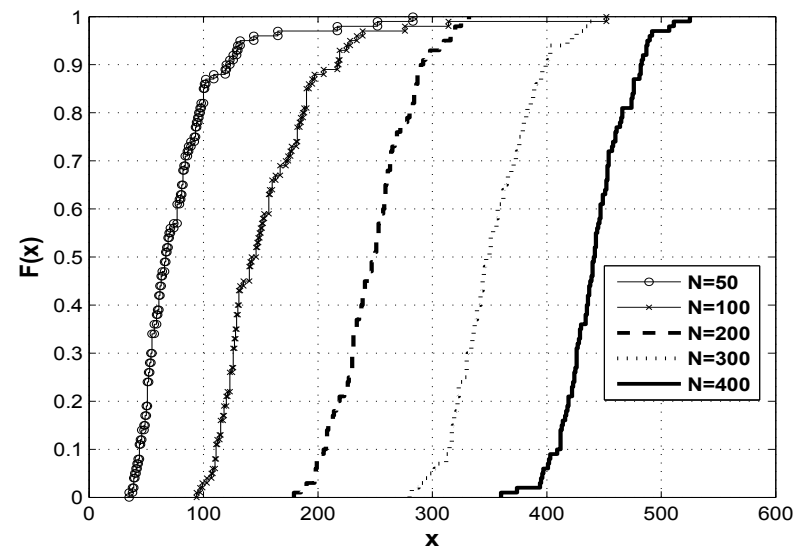

Figure 4: Empirical CDFs of the convergence time for different $N$, with $l_{N}=10$.

sum-rate of the resulting approximate NE is asymptotically approximately close to the optimal, and so is the minimal rate. This stems from the submartingality of the sum-rate process that our dynamics induce on the strategy profiles. This approach also enables a simple analysis of the convergence time. Simulations support our results and show convergence to an approximate PNE that only requires a small number of updates from each player. This also implies that our dynamics are inherently robust to the addition of new players. If, at some point in time, a new player arrives and chooses a channel, it likely requires a small portion of players to change their channel until an $\varepsilon$-PNE is maintained.

Our solution achieves self-configuration and low energy consumption, both of which are desirable for small, simple devices that will take part in the IoT and futuristic WSAN. Furthermore, such devices will require simple protocols, so the distributed fashion of our resource allocation scheme is of crucial value.

Last but not least, using probabilistic tools, by imposing a probability space over the set of games, is a strong tool to analyze the dynamics of a large class of games. This is a novel approach which can have many other new applications. 


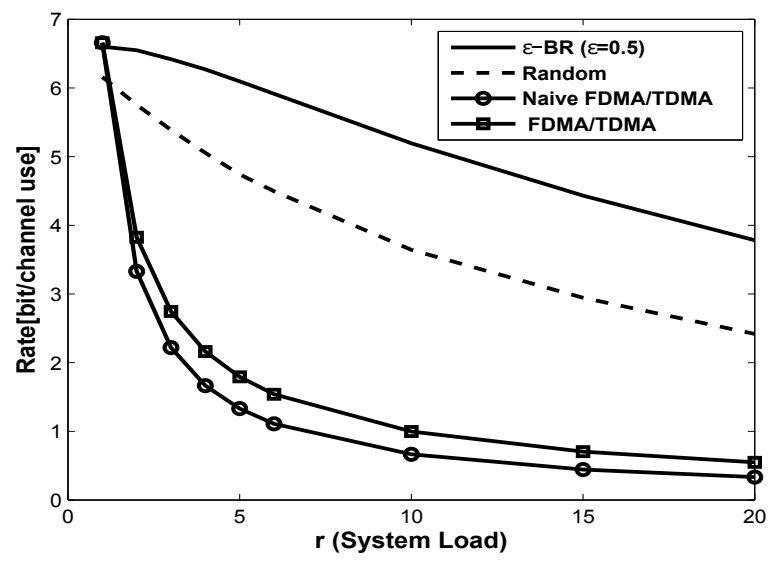

Figure 5: Mean rate for different $l_{N}$, with $N=300$.
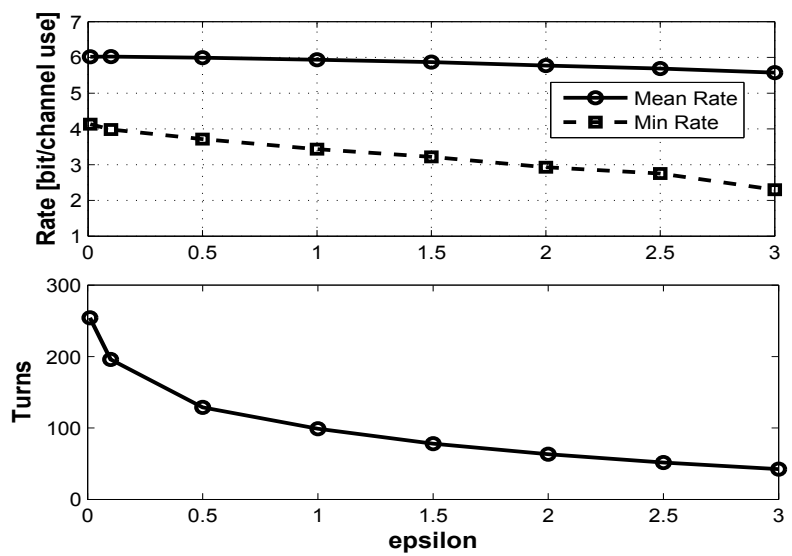

Figure 6: Rates and convergence time for different $\varepsilon$.

\section{APPENDiX A}

Proof of Lemma 3

Proof: Let player $n$ be the current acting player and denote $N_{\mathrm{far}, n}=\left|\mathcal{N}_{\mathrm{far}, n}\right|$ (see Definition 8 ). For some $q>1$, define the set

$$
\mathcal{K}_{g, n}\left(\mathbf{a}_{-n}\right)=\left\{k \mid a_{m} \neq k, \forall m \notin \mathcal{N}_{\text {far }, n}, N_{k}\left(\mathbf{a}_{-n}\right)<\left\lceil q l_{N}\right\rceil\right\}
$$

where $N_{k}\left(\mathbf{a}_{-n}\right)$ is the number of players (apart from player $n$ ) in channel $k$. This is the set of channels that contain only far players (from the receiver) and no more than $\left\lceil q l_{N}\right\rceil-1$ players (apart from $n$ ). We want to lower bound the cardinality of this set for all strategy profiles. Players that are not far can occupy at most $N-N_{\text {far }, n}$ channels, so each of them can reduce $\left|\mathcal{K}_{g, n}\left(\mathbf{a}_{-n}\right)\right|$ by at most one. Now we are left with $N_{\text {far }, n}$ far players and we need at least $\left\lceil q l_{N}\right\rceil$ of them to reduce $\left|\mathcal{K}_{g, n}\left(\mathbf{a}_{-n}\right)\right|$ by one, so

$$
\left|\mathcal{K}_{g, n}\left(\mathbf{a}_{-n}\right)\right| \geq K-\left(N-N_{\mathrm{far}, n}\right)-\left\lfloor\frac{N_{\mathrm{far}, n}}{\left\lceil q l_{N}\right\rceil}\right\rfloor \underset{(a)}{\geq} K+N_{\mathrm{far}, n}\left(1-\frac{1}{q l_{N}}\right)-N
$$


where in (a) we used the trivial inequality $\left\lfloor\frac{N_{\mathrm{far}, n}}{\left\lceil q l_{N}\right\rceil}\right\rfloor \leq \frac{N_{\mathrm{far}, n}}{q l_{N}}$. By Lemma 1

$$
\lim _{N \rightarrow \infty} \operatorname{Pr}\left(K+\left(1-\frac{1}{q l_{N}}\right) \min _{n \in \mathcal{N}} N_{\text {far }, n}-N \geq \frac{1}{2 l_{N}}\left(1-\frac{1}{q}\right) N\right)=\lim _{N \rightarrow \infty} \operatorname{Pr}\left(\min _{n \in \mathcal{N}} N_{\text {far }, n} \geq\left(1-\frac{1}{2} \frac{q-1}{q l_{N}-1}\right) N\right)=1
$$

In words, the probability that for each $n$ and a there exist at least $\frac{1}{2 l_{N}}\left(1-\frac{1}{q}\right) N$ channels in $\mathcal{K}_{g, n}\left(\mathbf{a}_{-n}\right)$ goes to 1 as $N \rightarrow \infty$. Now observe that for each $n$, each channel $k \in \mathcal{K}_{g, n}\left(\mathbf{a}_{-n}\right)$ satisfies

$$
I_{n}\left(k, \mathbf{a}_{-n}\right)=\sum_{\left\{m \mid a_{m}=k\right\}} g_{m, d(n)} P_{m} \underset{(a)}{\leq} \sum_{\left\{m \mid a_{m}=k\right\}} \frac{G P_{m}}{r_{m, d(n)}^{\alpha}} \leq \frac{\lambda^{\frac{\alpha}{2}} G q P_{0}}{\left(\frac{1}{2 \theta_{R}} \frac{q-1}{q l_{N}-1}\right)^{\alpha / 2}} l_{N}\left(\frac{\log N}{N}\right)^{\frac{\alpha}{2}}
$$

where (a) and (b) are for the worst case where all the involved indicator functions (see (1)) are equal to 1 and $r_{m, d(n)} \geq$ $\sqrt{\frac{1}{2 \theta_{R} \lambda} \frac{q-1}{q l_{N}-1}}$ (otherwise $g_{m, d(n)}=0$ ). Inequality (b) also uses $P_{m} \leq P_{0}\left(\frac{\log N}{N}\right)^{\frac{\alpha}{2}}$ and the fact that there are no more than $\left\lceil q l_{N}\right\rceil-1 \leq q l_{N}$ players in each of these channels. Denote $\gamma=\max _{n \in \mathcal{N}} \frac{\frac{g_{n, d(n)} P_{n}}{N_{0}}}{\frac{g_{n, d(n)} P_{n}}{N_{0}}+1}$. For every $k \in \mathcal{K}_{g, n}\left(\mathbf{a}_{-n}\right)$ we have

$$
\max _{n \in \mathcal{N}}\left|u_{n}\left(k, \mathbf{a}_{-n}\right)-\log _{2}\left(1+\frac{g_{n, d(n)} P_{n}}{N_{0}}\right)\right| \leq \max _{(a)} \frac{\gamma I_{n}\left(k, \mathbf{a}_{-n}\right)}{N_{0} \ln (2)} \leq \frac{\gamma \lambda^{\frac{\alpha}{2}} G q l_{N}}{\ln (2)\left(\frac{1}{2 \theta_{R}} \frac{q-1}{q l_{N}-1}\right)^{\alpha / 2}} \frac{P_{0}}{N_{0}}\left(\frac{\log N}{N}\right)^{\frac{\alpha}{2}}
$$

where (a) follows from Lemma 2 and (b) from (45). Since $B R_{n}(\mathbf{a})$ (the exact BR) has better utility than any of $k \in \mathcal{K}_{g, n}\left(\mathbf{a}_{-n}\right)$, (24) follows immediately. Since $\frac{l_{N}}{\left(\frac{q-1}{q l_{N}-1}\right)^{\alpha / 2}}\left(\frac{\log N}{N}\right)^{\frac{\alpha}{2}} \rightarrow 0$ as $N \rightarrow \infty$, we conclude that for a large enough $N$ (such that (46) is smaller than $\left.\varepsilon+\log _{2}\left(1+\frac{g_{n, d(n)} P_{n}}{N_{0}}\right)-u\left(B R_{n}(\mathbf{a})\right)\right)$ we obtain that each $k \in \mathcal{K}_{g, n}\left(\mathbf{a}_{-n}\right)$ is in $B_{\varepsilon}\left(\mathbf{a}_{-n}\right)$, i.e., $\mathcal{K}_{g, n}\left(\mathbf{a}_{-n}\right) \subseteq$ $B_{\varepsilon}\left(\mathbf{a}_{-n}\right)$ for each $n$ and $\mathbf{a}$. This fact together with (43), (44) yield $\lim _{N \rightarrow \infty} \operatorname{Pr}\left(\forall \mathbf{a}, \min _{n \in \mathcal{N}}\left|B_{\varepsilon}\left(\mathbf{a}_{-n}\right)\right| \geq \frac{1}{2 l_{N}}\left(1-\frac{1}{q}\right) N\right)=1$. Note that our analysis is the same regardless of the strategy profile (hence also $t$ ) and depends solely on the players' locations.

\section{APPENDIX B}

\section{Proof of LEMMA 4}

Proof: Denote $N_{\text {near }, n}=\left|\mathcal{N}_{\text {near }, n}\right|$ and $\rho=\left(\frac{\log N}{N}\right)^{\frac{\alpha}{2 \alpha+4}}$ (see Definition $[$ ). Let $\varepsilon>0$. By the definition of the approximate BR dynamics, for each $n$ and $\mathbf{a}_{t}$ such that $\mathbf{a}_{\mathbf{t}+\mathbf{1}}=B R_{\varepsilon, n}\left(\mathbf{a}_{\mathbf{t}}\right) \neq \mathbf{a}_{t}$

$$
E_{n}\left\{R_{n}\left(\mathbf{a}_{\mathbf{t}+\mathbf{1}}\right)-R_{n}\left(\mathbf{a}_{\mathbf{t}}\right) \mid \mathbf{a}_{\mathbf{t}}\right\} \geq \frac{\varepsilon}{2}
$$

with probability 1 with respect to the random game. Denote the rate decrease caused to player $m$ by the fact that player $n$ moves to his channel as

$$
\triangle R_{m}\left(\mathbf{a}_{\mathbf{t}+\mathbf{1}}\right)=\triangle R_{m}\left(a_{m}, \mathbf{a}_{-n, t}\right)=R\left(I_{m}\left(\mathbf{a}_{\mathbf{t}}\right)+g_{n, d(m)} P_{n}\right)-R\left(I_{m}\left(\mathbf{a}_{\mathbf{t}}\right)\right)
$$

where $I_{m}\left(\mathbf{a}_{\mathbf{t}}\right)$ is his current interference, and $g_{n, d(m)} P_{n}$ is the additional interference caused by player $n$. For each $m$ such that $m \notin \mathcal{N}_{\text {near }, n}$ and $d(m) \neq n$ we have

$$
g_{n, d(m)} \leq \frac{G}{(a)} \frac{\pi^{\frac{\alpha}{2}} \lambda^{\frac{\alpha}{2}} G}{r^{\alpha}, d(m)}
$$


where (a) and (b) are for the worst case where the indicator functions in (1) are equal to 1 and $r_{n, d(m)} \geq \frac{\rho}{\sqrt{\pi \lambda}}$, otherwise $g_{n, d(m)}=0$. Denote $\gamma=\max _{n \in \mathcal{N}} \frac{\frac{g_{n, d(n)} P_{n}}{N_{0}}}{\frac{g_{n, d(n)} P_{n}}{N_{0}}+1}$ and observe that

$$
\triangle R_{m}\left(a_{m}, \mathbf{a}_{-n, t}\right) \underset{(a)}{\geq}-\frac{\gamma}{\ln (2)} \frac{I_{m}\left(a_{m}, \mathbf{a}_{-n, t}\right)-I_{m}\left(\mathbf{a}_{t}\right)}{N_{0}+I_{m}\left(\mathbf{a}_{t}\right)} \underset{(b)}{\geq}-\frac{\gamma}{\ln (2)} \frac{g_{n, d(m)} P_{n}}{N_{0}} \underset{(c)}{\geq}-\frac{\gamma \pi^{\frac{\alpha}{2}} \lambda^{\frac{\alpha}{2}} G}{\ln (2) \rho^{\alpha}} \frac{P_{0}}{N_{0}}\left(\frac{\log N}{N}\right)^{\frac{\alpha}{2}}
$$

where (a) is from (2), (48) and Lemma 2, (b) follows from $I_{m}\left(\mathbf{a}_{t}\right) \geq 0$ and (c) is from (49) together with $P_{n} \leq P_{0}\left(\frac{\log N}{N}\right)^{\frac{\alpha}{2}}$.

For each $m \in \mathcal{N}_{\text {near, } n}$ and also for each $m$ such that $d(m)=n$ we have

$$
\triangle R_{m}\left(a_{m}, \mathbf{a}_{-n, t}\right) \geq-\max _{m \in \mathcal{N}} R\left(I_{m}\left(\mathbf{a}_{\mathbf{t}}\right)\right) \geq-\max _{m \in \mathcal{N}} \log _{2}\left(1+\frac{g_{m, d(m)} P_{m}}{N_{0}}\right)
$$

Denote $C_{\max }=\max _{m \in \mathcal{N}} \log _{2}\left(1+\frac{g_{m, d(m)} P_{m}}{N_{0}}\right)$ and $\mathcal{N}_{\text {bad }, n}=\mathcal{N}_{\text {near }, n} \bigcup\{m \mid m \in \mathcal{N}, d(m)=n\} 2$. If indeed $\left|B_{\frac{\varepsilon}{2}}\left(\mathbf{a}_{-n, t}\right)\right| \geq$ $\frac{1}{2 l_{N}}\left(1-\frac{1}{q}\right) N$ for some $q>1$ and $N_{\text {near }, n} \leq \frac{\theta_{T}}{\pi} \rho^{2} N$ then for each $n$ and $\mathbf{a}_{t}$ such that $\mathbf{a}_{\mathbf{t}+\mathbf{1}}=B R_{\varepsilon, n}\left(\mathbf{a}_{\mathbf{t}}\right) \neq \mathbf{a}_{t}$ we obtain

$$
\begin{aligned}
& E_{n}\left\{\sum_{\left\{m \mid a_{n, t+1}=a_{m, t}\right\}} \triangle R_{m}\left(a_{n, t+1}, \mathbf{a}_{-n, t}\right) \mid \mathbf{a}_{\mathbf{t}}\right\} \underset{(a)}{=} \frac{1}{\left|B_{\frac{\varepsilon}{2}}\right|} \sum_{k \in B_{\frac{\varepsilon}{2}}} \sum_{m \mid a_{m, t}=k} \Delta R_{m}\left(k, \mathbf{a}_{-n, t}\right) \underset{(b)}{=} \\
& \frac{1}{\left|B_{\frac{\varepsilon}{2}}\right|} \sum_{m \mid a_{m, t} \in B_{\frac{\varepsilon}{2}}} \triangle R_{m}\left(a_{m}, \mathbf{a}_{-n, t}\right) \geq \frac{1}{\left|B_{\frac{\varepsilon}{2}}\right|} \sum_{m \in \mathcal{N}} \triangle R_{m}\left(a_{m}, \mathbf{a}_{-n, t}\right)= \\
& \frac{1}{\left|B_{\frac{\varepsilon}{2}}\right|} \sum_{m \notin \mathcal{N}_{\text {bad }, n}} \triangle R_{m}\left(a_{m}, \mathbf{a}_{-n, t}\right)+\frac{1}{\left|B_{\frac{\varepsilon}{2}}\right|} \sum_{m \in \mathcal{N}_{\text {bad }, n}} \Delta R_{m}\left(a_{m}, \mathbf{a}_{-n, t}\right) \underset{(c)}{\geq} \\
& -\frac{1}{\left|B_{\frac{\varepsilon}{2}}\right|}\left(\frac{\gamma \pi^{\frac{\alpha}{2}} \lambda^{\frac{\alpha}{2}} G}{\ln (2) \rho^{\alpha}} \frac{P_{n}}{N_{0}} \sum_{m \notin \mathcal{N}_{\text {bad }, n}} 1+C_{\max } \sum_{m \in \mathcal{N}_{\text {bad }, n}} 1\right) \geq-\frac{1}{\left|B \frac{\varepsilon}{2}\right|}\left(\frac{\gamma \pi^{\frac{\alpha}{2}} \lambda^{\frac{\alpha}{2}} G}{\ln (2) \rho^{\alpha}} \frac{P_{n}}{N_{0}} N+C_{\text {max }}\left(N_{\text {near }, n}+S\right)\right) \underset{(\bar{d})}{\geq} \\
& -\frac{2 l_{N}}{1-\frac{1}{q}}\left(\frac{P_{0}}{N_{0}} \frac{\gamma \pi^{\frac{\alpha}{2}} \lambda^{\frac{\alpha}{2}} G}{\ln (2)} \rho^{-\alpha}\left(\frac{\log N}{N}\right)^{\frac{\alpha}{2}}+C_{\max }\left(\frac{\theta_{T}}{\pi} \rho^{2}+\frac{S}{N}\right)\right)
\end{aligned}
$$

where we omitted $\mathbf{a}_{-n, t}$ from $B_{\frac{\varepsilon}{2}}\left(\mathbf{a}_{-n, t}\right)$ for convenience. Equality (a) follows from the expectation definition and (b) from changing the order of summation. Inequality (c) follows from (50) and (51) and (d) from $\left|B_{\frac{\varepsilon}{2}}\left(\mathbf{a}_{-n, t}\right)\right| \geq \frac{1}{2 l_{N}}\left(1-\frac{1}{q}\right) N$ and $N_{\text {near }, n} \leq \frac{\theta_{T}}{\pi} \rho^{2} N$

By Lemma 1 and Lemma 3. $\max _{n \in \mathcal{N}} N_{\text {near, } n} \leq \frac{\theta_{T}}{\pi} \rho^{2} N$ and $\min _{n \in \mathcal{N}}\left|B_{\frac{\varepsilon}{2}}\left(\mathbf{a}_{-n, t}\right)\right| \geq \frac{1}{2 l_{N}}\left(1-\frac{1}{q}\right) N$ occur with probability that approaches 1 as $N \rightarrow \infty$. This implies that inequality (d) in (52) and hence all (52) is true for each $n$ with probability that approaches 1 as $N \rightarrow \infty$. From (52) it is clear that since $\frac{P_{0}}{N_{0}} \frac{\gamma \pi^{\frac{\alpha}{2}} \lambda^{\frac{\alpha}{2}} G}{\ln (2)}\left(\frac{\log N}{N}\right)^{\frac{\alpha}{2}}$ is vanishing, the optimal value of $\rho$ that minimizes the expression within the brackets is also vanishing to lower the term $\frac{\theta_{T}}{\pi} \rho^{2} C_{\max }$. This accounts for the choice of $\rho=\left(\frac{\log N}{N}\right)^{\frac{\alpha}{2 \alpha+4}}$. Substituting it and adding (47) and 52 we get that the probability that for every and $n$ such that $\mathbf{a}_{\mathbf{t}+\mathbf{1}}=B R_{\varepsilon, n}\left(\mathbf{a}_{\mathbf{t}}\right) \neq \mathbf{a}_{t}$, and all $t \geq 0$

$$
E_{n}\left\{\Delta_{1}\left(\mathbf{a}_{\mathbf{t}}\right)+\Delta_{2}\left(\mathbf{a}_{\mathbf{t}}\right) \mid \mathbf{a}_{\mathbf{t}}\right\} \geq \frac{\varepsilon}{2}-\frac{2 l_{N}}{1-\frac{1}{q}}\left(\frac{\log N}{N}\right)^{\frac{\alpha}{\alpha+2}}\left(\frac{P_{0}}{N_{0}} \frac{\gamma \pi^{\frac{\alpha}{2}} \lambda^{\frac{\alpha}{2}} G}{\ln (2)}+C_{\max }\left(\frac{\theta_{T}}{\pi}+\frac{S}{N^{\frac{2}{\alpha+2}}}\right)\right)>0
$$

approaches 1 as $N \rightarrow \infty$.

\footnotetext{
${ }^{2}$ Player $n$ as a transmitter is "near" himself as a destination for other players (as many as $S$ ). We take into account the possibility that player $n$ cannot cancel his own transmission, so $g_{n, n}$ is infinite.
} 


\section{REFERENCES}

[1] I. Bistritz and A. Leshem, "Convergence of approximate best-response dynamics in interference games," in Decision and Control (CDC), 2016 IEEE 55th Conference on, 2016, pp. 4433-4438.

[2] Z. Han, Game theory in wireless and communication networks: theory, models, and applications. Cambridge University Press, 2012.

[3] E. G. Larsson, E. Jorswieck, J. Lindblom, R. Mochaourab et al., "Game theory and the flat-fading Gaussian interference channel," Signal Processing Magazine, IEEE, vol. 26, no. 5, pp. 18-27, 2009.

[4] W. Saad, Z. Han, H. V. Poor, and T. Başar, "Game-theoretic methods for the smart grid: An overview of microgrid systems, demand-side management, and smart grid communications," Signal Processing Magazine, IEEE, vol. 29, no. 5, pp. 86-105, 2012.

[5] A. Leshem and E. Zehavi, "Game theory and the frequency selective interference channel," IEEE Signal Processing Magazine, vol. 26, no. 5, pp. 28-40, 2009.

[6] A. Laufer and A. Leshem, "Distributed coordination of spectrum and the prisoner s dilemma," in New Frontiers in Dynamic Spectrum Access Networks, 2005. DySPAN 2005. 2005 First IEEE International Symposium on, 2005, pp. 94-100.

[7] J. Nash, "Non-cooperative games," The Annals of Mathematics, vol. 54, no. 2, pp. 286-295, 1951.

[8] E. Altman, K. Avrachenkov, I. Menache, G. Miller, B. J. Prabhu, and A. Shwartz, "Dynamic discrete power control in cellular networks," IEEE Transactions on Automatic Control, vol. 54, no. 10, pp. 2328-2340, 2009.

[9] E. Arcaute, R. Johari, and S. Mannor, "Network formation: Bilateral contracting and myopic dynamics," IEEE Transactions on Automatic Control, vol. 54 , no. 8 , pp. $1765-1778,2009$.

[10] O. N. Gharehshiran, V. Krishnamurthy, and G. Yin, "Adaptive search algorithms for discrete stochastic optimization: A smooth best-response approach," IEEE Transactions on Automatic Control, vol. 62, no. 1, pp. 161-176, 2017.

[11] M. Goemans, V. Mirrokni, and A. Vetta, "Sink equilibria and convergence," in Foundations of Computer Science, 2005. FOCS 2005. 46th Annual IEEE Symposium on, 2005, pp. 142-151.

[12] A. Fabrikant, A. D. Jaggard, and M. Schapira, "On the structure of weakly acyclic games," in International Symposium on Algorithmic Game Theory, 2010, pp. 604-612.

[13] H. P. Young, "The evolution of conventions," Econometrica: Journal of the Econometric Society, vol. 61, no. 1, pp. 57-84, 1993.

[14] V. S. Mirrokni and A. Skopalik, "On the complexity of Nash dynamics and sink equilibria," in Proceedings of the 10th ACM conference on Electronic commerce, 2009, pp. 1-10.

[15] D. Monderer and L. S. Shapley, "Potential games," Games and economic behavior, vol. 14, no. 1, pp. 124-143, 1996.

[16] J. R. Marden, H. P. Young, G. Arslan, and J. S. Shamma, "Payoff-based dynamics for multiplayer weakly acyclic games," SIAM Journal on Control and Optimization, vol. 48, no. 1, pp. 373-396, 2009.

[17] E. Altman, A. Kumar, and Y. Hayel, "A potential game approach for uplink resource allocation in a multichannel wireless access network," in Proceedings of the Fourth International ICST Conference on Performance Evaluation Methodologies and Tools, 2009, p. 72.

[18] K. Cohen, A. Leshem, and E. Zehavi, "Game theoretic aspects of the multi-channel ALOHA protocol in cognitive radio networks," Selected Areas in Communications, IEEE Journal on, vol. 31, no. 11, pp. 2276-2288, 2013.

[19] G. Scutari, S. Barbarossa, and D. P. Palomar, "Potential games: A framework for vector power control problems with coupled constraints," in Acoustics, Speech and Signal Processing, 2006. ICASSP 2006 Proceedings. 2006 IEEE International Conference on, vol. 4, 2006, pp. IV-IV.

[20] A. Cortés and S. Martínez, "Self-triggered best-response dynamics for continuous games," IEEE Transactions on Automatic Control, vol. 60, no. 4, pp. 1115-1120, 2015.

[21] J. R. Marden and A. Wierman, "Overcoming the limitations of utility design for multiagent systems," IEEE Transactions on Automatic Control, vol. 58, no. 6, pp. 1402-1415, 2013.

[22] A. Fabrikant, C. Papadimitriou, and K. Talwar, "The complexity of pure Nash equilibria," in Proceedings of the thirty-sixth annual ACM symposium on Theory of computing, 2004, pp. 604-612.

[23] S. Chien and A. Sinclair, "Convergence to approximate Nash equilibria in congestion games," in Proceedings of the eighteenth annual ACM-SIAM symposium on Discrete algorithms, 2007, pp. 169-178. 
[24] M. Cesana, I. Malanchini, and A. Capone, "Modelling network selection and resource allocation in wireless access networks with non-cooperative games," in Mobile Ad Hoc and Sensor Systems, 2008. MASS 2008. 5th IEEE International Conference on, 2008, pp. 404-409.

[25] E. Anshelevich, A. Dasgupta, J. Kleinberg, E. Tardos, T. Wexler, and T. Roughgarden, "The price of stability for network design with fair cost allocation," SIAM Journal on Computing, vol. 38, no. 4, pp. 1602-1623, 2008.

[26] J. R. Marden and T. Roughgarden, "Generalized efficiency bounds in distributed resource allocation," IEEE Transactions on Automatic Control, vol. 59, no. 3, pp. 571-584, 2014.

[27] C. Tekin, M. Liu, R. Southwell, J. Huang, and S. H. A. Ahmad, "Atomic congestion games on graphs and their applications in networking," Networking, IEEE/ACM Transactions on, vol. 20, no. 5, pp. 1541-1552, 2012.

[28] O. Candogan, A. Ozdaglar, and P. A. Parrilo, "Near-potential games: Geometry and dynamics," ACM Transactions on Economics and Computation, vol. 1 , no. 2 , p. 11, 2013 .

[29] — - "Dynamics in near-potential games," Games and Economic Behavior, vol. 82, pp. 66-90, 2013.

[30] E. Altman, T. Başar, and R. Srikant, "Nash equilibria for combined flow control and routing in networks: Asymptotic behavior for a large number of users," IEEE Transactions on Automatic Control, vol. 47, no. 6, pp. 917-930, 2002.

[31] S. Grammatico, F. Parise, M. Colombino, and J. Lygeros, "Decentralized convergence to Nash equilibria in constrained mean field control," IEEE Transactions on Automatic Control, vol. 61, no. 11, pp. 3315-3329, 2016.

[32] H. Tembine, Q. Zhu, and T. Başar, "Risk-sensitive mean-field games," IEEE Transactions on Automatic Control, vol. 59, no. 4, pp. 835-850, 2014.

[33] W. H. Sandholm, Population games and evolutionary dynamics, 2010, Ed. MIT press, 2010.

[34] E. Altman and Y. Hayel, "Markov decision evolutionary games," IEEE Transactions on Automatic Control, vol. 55, no. 7, pp. 1560-1569, 2010.

[35] D. Madeo and C. Mocenni, "Game interactions and dynamics on networked populations," IEEE Transactions on Automatic Control, vol. 60, no. 7, pp. $1801-1810,2015$

[36] A. Leshem and E. Zehavi, "Cooperative game theory and the Gaussian interference channel," Selected Areas in Communications, IEEE Journal on, vol. 26 , no. 7, pp. 1078-1088, 2008.

[37] I. Bistritz and A. Leshem, "Asymptotically optimal distributed channel allocation: a competitive game-theoretic approach," in Communication, Control, and Computing (Allerton), 2015 53nd Annual Allerton Conference on, 2015, pp. 1272-1279.

[38] S. T. Chung, S. J. Kim, J. Lee, and J. M. Cioffi, "A game-theoretic approach to power allocation in frequency-selective Gaussian interference channels," in in Proc. IEEE International Symposium on Inform. Theory, Pacifico, 2003, p. 316.

[39] Z. Chen, S. A. Vorobyov, C.-X. Wang, and J. Thompson, "Pareto region characterization for rate control in MIMO interference systems and Nash bargaining," IEEE Transactions on Automatic Control, vol. 57, no. 12, pp. 3203-3208, 2012.

[40] R. W. Rosenthal, "A class of games possessing pure-strategy Nash equilibria," International Journal of Game Theory, vol. 2, no. 1, pp. 65-67., 1973.

[41] P. Gupta and P. R. Kumar, "The capacity of wireless networks," IEEE Transactions on Information Theory, vol. 46, no. 2, pp. 388-404, 2000.

[42] M. Grossglauser and D. Tse, "Mobility increases the capacity of ad-hoc wireless networks," in INFOCOM 2001. Twentieth Annual Joint Conference of the IEEE Computer and Communications Societies. Proceedings. IEEE, 2001, pp. 1360-1369.

[43] P. Gupta and P. R. Kumar, "Critical power for asymptotic connectivity in wireless networks," in Stochastic analysis, control, optimization and applications, 1999, pp. 547-566.

[44] J. Díaz, J. Petit, and M. Serna, "A random graph model for optical networks of sensors," IEEE Transactions on Mobile Computing, vol. 2, no. 3, pp. 186-196, 2003

[45] S. G. Kiani and D. Gesbert, "Optimal and distributed scheduling for multicell capacity maximization," IEEE Transactions on Wireless Communications, vol. 7, no. 1, pp. 288-297, 2008 .

[46] L. Breiman, Probability, volume 7 of Classics in Applied Mathematics. Society for Industrial and Applied Mathematics (SIAM), Philadelphia, PA, 1992.

[47] J. Ni, R. Srikant, and X. Wu, "Coloring spatial point processes with applications to peer discovery in large wireless networks," IEEE/ACM Transactions on Networking (TON), vol. 19, no. 2, pp. 575-588, 2011.

[48] N. Alon and J. H. Spencer, The probabilistic method. John Wiley \& Sons, 2015. 